Review

\title{
The Structure of Urban Flows
}

\author{
Pablo Torres ${ }^{1}$, Soledad Le Clainche ${ }^{2}$ and Ricardo Vinuesa ${ }^{1, *}$ \\ 1 SimEx/FLOW, Engineering Mechanics, KTH Royal Institute of Technology, SE-100 44 Stockholm, Sweden \\ 2 School of Aerospace Engineering, Universidad Politécnica de Madrid, 28040 Madrid, Spain \\ * Correspondence: rvinuesa@mech.kth.se
}

Version September 23, 2020 submitted to Preprints

1 Abstract: Understanding the flow in urban environments is an increasingly relevant problem due to 2 its significant impact on air quality and thermal effects in cities worldwide. In this review we provide 3 an overview of efforts based on experiments and simulations to gain insight into this complex physical 4 phenomenon. We highlight the relevance of coherent structures in urban flows, which are responsible 5 for the pollutant-dispersion and thermal fields in the city. We also suggest a more widespread use of 6 data-driven methods to characterize flow structures as a way to further understand the dynamics of 7 urban flows, with the aim of tackling the important sustainability challenges associated to them.

s Keywords: urban flows; coherent structures; experiments; simulations; data-driven methods

\section{Motivation}

Urban areas are key elements in our society: around 75\% of the population currently lives in cities in the European Union (EU) and it is expected that, by 2050, 7 out of 10 people will be urban dwellers worldwide [1]. Cities are responsible for a significant fraction of the total greenhouse emissions in the world (above 60\% according to Ref. [2]), and they are expected to play a prominent role towards the achievement of the climate-change targets from the Paris Agreement [3]. Two of the main challenges from cities for the coming years are the levels of air pollution and the so-called urban-heat-island (UHI) effect. According to the European Environment Agency (EEA), around 90\% of the urban population in the EU was exposed to air pollution levels exceeding the ones recommended by the World Health Organization (WHO) between 2014 and 2016, and those levels of air pollution were responsible for around 500,000 premature deaths in 2015 [4]. In fact, more recent estimates indicate that this number may be as high as 800,000 , where $40-80 \%$ of the cases are due to cardiovascular events [5]. On the other hand, the UHI phenomenon (whereby the temperatures in cities are higher than in the surrounding rural areas [6]) was related to 70,000 deaths due to the heat wave in Europe during the summer of 2003 [7].

Although the EU has introduced the use of predictive models to support pollutant-concentration measurements [8], currently available techniques are unable to provide the spatial and temporal accuracy required to reproduce the pollutant-dispersion patterns within urban environments [9]. Similarly, more robust remote-sensing methods need to be developed in order to accurately predict the heat fluxes relevant to UHI phenomena [10]. Therefore, there is a pressing need for improved prediction and assessment methods to tackle these challenges and enable urban sustainability in the near future. Furthermore, it is necessary to gain further insight into the physics responsible for the pollutant-concentration and thermal distributions within cities. In particular, the coherent structures present in the flow need to be studied in depth, so as to be able to develop effective strategies to mitigate these negative effects. This has important implications on the Sustainable Development Goals (SDGs) 11 (on sustainable cities and communities) and 13 (on climate change) from the United Nations (UN) 2030 Agenda [11,12].

This study presents an extensive review on the numerical and experimental work that has been developed over several decades to analyze urban flows. The review introduces some examples based on simple configurations, using squared blocks modelling buildings, which help to establish the fundamental physics of this type of problem, and also presents more complex and realistic scenarios 
providing specific ideas of the pollution dispersion in real cities. The identification of coherent structures plays a mayor role in modelling urban flows, and this approach has been barely explored due to the high complexity of the problem. Using new data-driven modal-decomposition techniques will shed light into the mechanisms producing urban pollution, and will present new solutions for the cities of the future.

This article is structured as follows: in $\$ 2$ we provide a review of the fundametnal mechanisms present in urban flows; in $\$ 3$ we give an overview of experimetnal campaigns aimed at understanding urban flows; on the other hand, $\S 4$ discusses a selection of numerical simulations of the flow in urban environments; an assessment of the relevance of coherent structures in urban flows is given in §5; an outlook of the field is provided in $\S 6$ and a selection of relevant modal decompositions to analyze urban flows is given in the Appendix.

\section{Fundamentals of urban flows}

Urban environments can be described in terms of different length scales [13]: from the larger regional and city scales (in the range from 10 to $200 \mathrm{~km}$ ), to the smaller neighborhood and street levels (between $100 \mathrm{~m}$ and $2 \mathrm{~km}$ ). The regional scale is affected by the urban area, and the flow responds to mean synoptic meteorological patterns perturbed by the city, as discussed by Britter and Hanna [13]. They also argue that at the city scale the "variations of the flow are mostly averaged out". On the other hand, most of the aspects affecting pedestrian comfort, air quality, emissions from vehicles, etc. need to be assessed at the street scale. In particular, the region of the flow directly affected by the obstacles is the urban-canopy sublayer [14]. Oke [15] provided an empirical description of the flow regimes visible in this layer, where three flow regimes are found depending on the ratio between the street width and the building height [16]: for narrow streets, the flow above the canopy can barely penetrate down to the street (skimming flow), and only one vortex is observed between the buildings; progressively wider streets lead first to the wake-interference flow and later to the isolated-roughness regime, which exhibit much more interaction with the flow above the roof level and significantly more complex physics. In Figure 1 we show an instantaneous flow visualization in a simplified urban environment consisting of 4 arrays of blocks equally spaced in the streamwise direction and 3 in the span. This $4 \times 3$ model was simulated through well-resolved LES, see more details in $\S 4$ and Ref. [17]. This figure illustrates the complex interactions of the vortical structures among the various arrays, together with the frame of reference considered for this work, as well as the geometrical parameters defining the urban environment. These parameters determine the regime observed in the urban area [15], in particular the width-to-height $b / h$ and the height-to-separation $h / \ell$ ratios. As shown in Figure 2, while $b / h$ has a small impact on the resulting regime (particularly for tall buildings), the most critical parameter is $h / \ell[15]$.

The instantaneous flow in the three regimes discussed by Oke [15] is shown in Figure 3, where we consider two wall-mounted obstacles with varying streamwise separation. In this figure, also obtained through well-resolved LES $[17,19]$, the flow is represented by vortical structures identified with the $\lambda_{2}$ method [18]. All the obstacles have $b / h=0.5$, and the various cases exhibit different values of $\ell$ leading to various interactions between the wake of the first obstacle and the leeward side of the second one. In Figure 3 (top) we can visualize the skimming-flow regime, obtained with $h / \ell=1$, in which the flow above the obstacles can barely penetrate in the region between the two blocks due to the very prominent effect of the first wake. A larger separation, leading to $h / \ell=0.5$, is observed in Figure 3 (middle), which illustrates the wake-interference regime. Although there is still some interaction between the wake of the first obstacle and the second block, it is clear that some momentum transport from the flow above the canopy will take place between both obstacles. Finally, the isolated-roughness regime is observed in Figure 3 (bottom), with $h / \ell=0.25$. Here the interaction between the two obstacles is limited, and therefore there will be significant momentum exchange between this region and the flow above the canopy. 


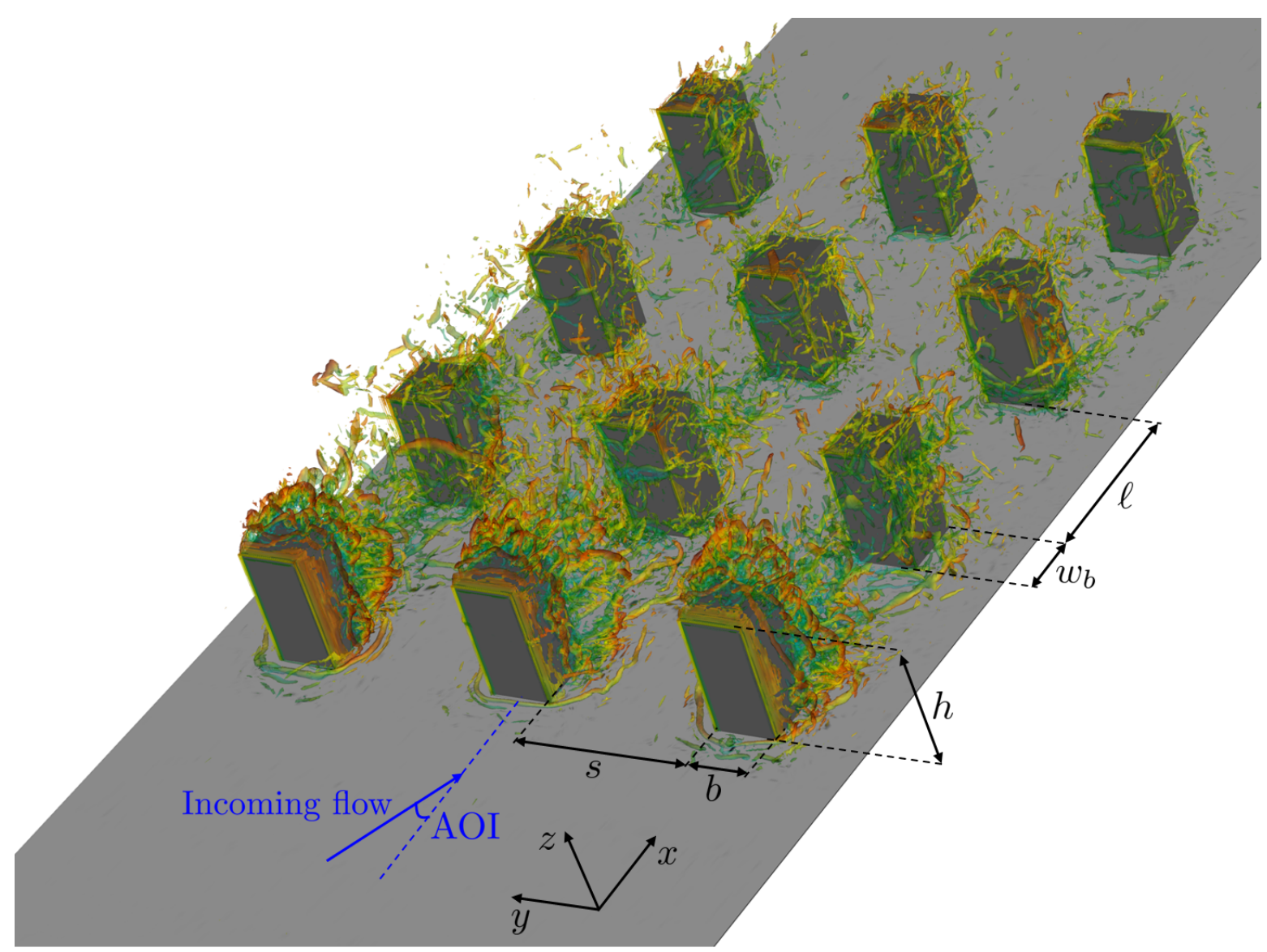

Figure 1. Simplified urban model, indicating the geometrical parameters and the definition of the angle of incidence (AOI). Instantaneous vortical structures identified with the $\lambda_{2}$ method [18] are shown with an isosurface of -40 (scaled in terms of the freestream velocity $U_{\infty}$ and $h$ ). The structures are colored by streamwise velocity, ranging from (dark blue) -1.2 to (dark red) 1.8. Light grey indicates the bottom wall, and dark grey the buildings.

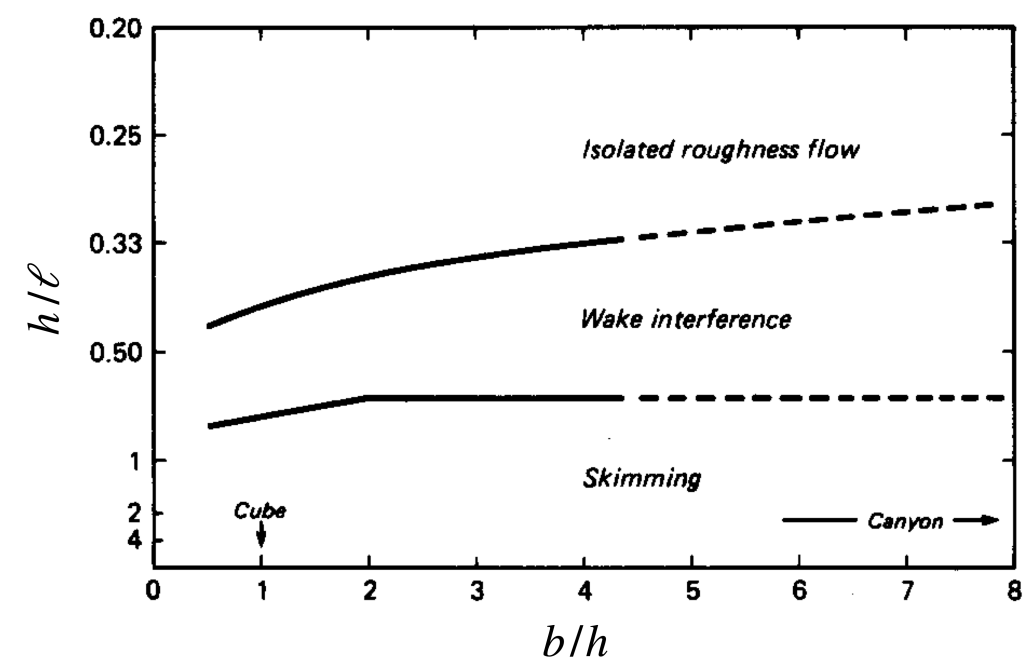

Figure 2. Dependence of the resulting flow regimes on the geometrical parameters shown in Figure 1. Adapted from Ref. [15], with permission of the publisher (Elsevier). 

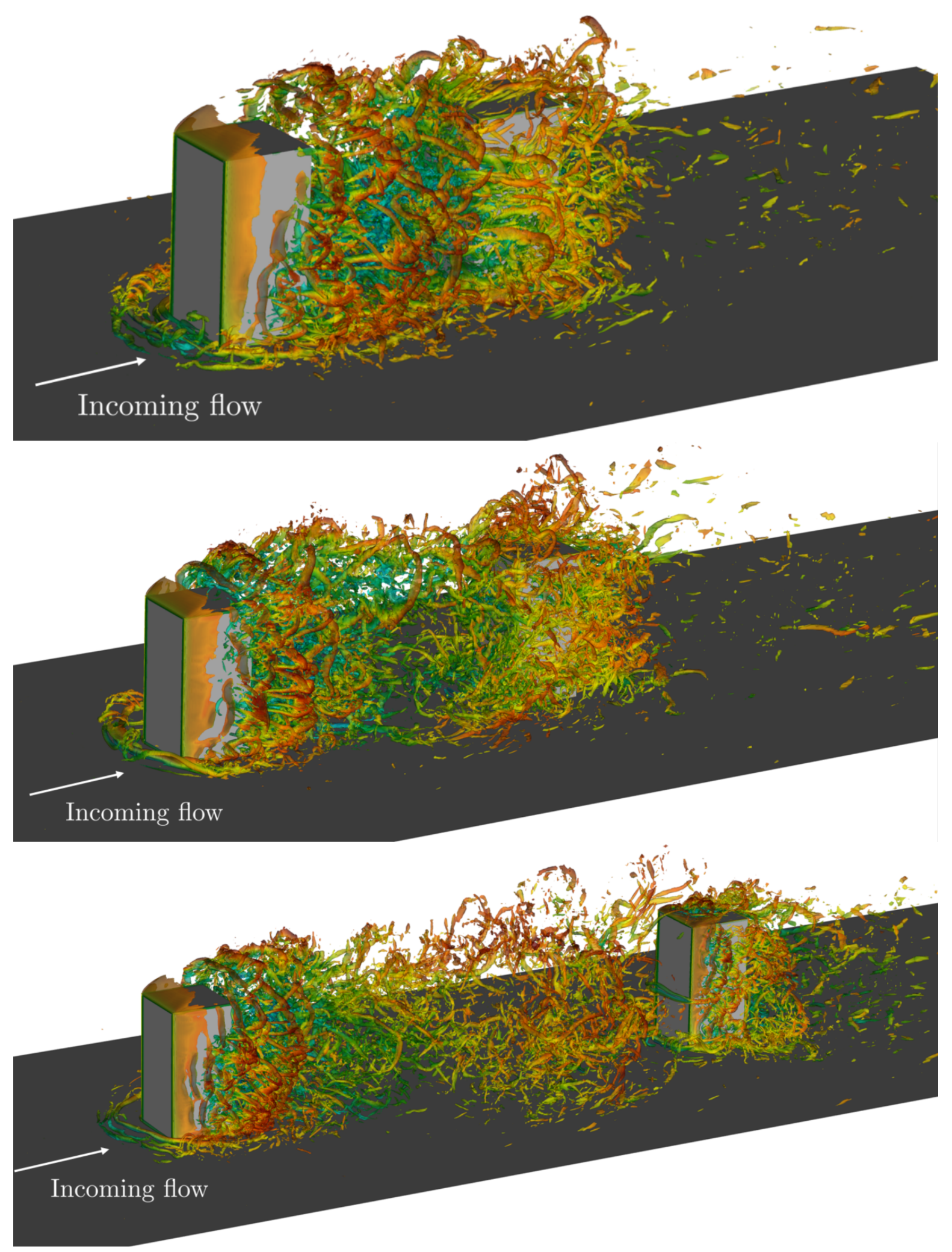

Figure 3. Instantaneous visualizations of the flow around two wall-mounted obstacles, showing vortical structures identified with the $\lambda_{2}$ method [18], with an isosurface of -40 (scaled in terms of $U_{\infty}$ and $h$ ). The flow exhibits three regimes depending on the obstacle separation, according to the classification by Oke [15]: (top) skimming flow, (middle) wake interference and (bottom) isolated roughness. The structures are colored by streamwise velocity, ranging from (dark blue) -1.2 to (dark red) 1.8 . 
The study of urban-flow regimes was expanded in the work of Zajic et al. [20], who the authors analysed flow behaviour in a central business district (CBD). This study extended the description of Oke [15] to account for three-dimensional effects. In fact, important effects are found in the vertical edges which are only visible when the spanwise direction is considered. In three dimensions, flow analysis is segregated into the streamwise and spanwise coordinates. In the streamwise direction, i.e. following the $x$ coordinate as shown in Figure 1, the length-to-height ratio $w_{b} / h$ is the principal factor affecting flow behaviour. For small values of $w_{b} / h$ the shear layer is completely detached from the obstacle. On the contrary, this ratio grows the flow tends to exhibit a greater attachment to the surface and edges of the obstacle. An equivalent analysis can be applied in the spanwise direction $y$, this time as a function of the width-to-height ratio $b / h$. As the obstacle height grows, the recirculation bubble behind the obstacle tends to be overwhelmed by the side separation layer, resulting in intense turbulent fluctuations which may destroy the separation bubble [20]. The separation of buildings in the spanwise direction $s$ changes the behaviour of the wake as well as the side-wall shear-layer interaction [20]. As the obstacles become closer in the spanwise direction, the wake-side-wall interaction increases. Additionally, the interaction changes the flow response to the surrounding physical factors. For instance, for an intermediate spanwise separation, the flow appears to be independent of the streamwise separation $\ell$, due to the fact that the adjacent obstacle in the streamwise direction induces a jet behaviour on the flow. This phenomenon supports the need of a three-dimensional approach in the study of urban flows. Zajic et al. [20] also discussed the effects of non-uniform obstacle heights on the flow. In fact, larger differences in height lead to increased vorticity. This phenomenon results in more prominent turbulent structures.

Other works propose alternative classifications of flow regimes within the urban environment. Britter and Hunt [21] define flow regimes in terms of the degree of interaction between the vortices and the freestream flow. According to Penwarden and Wise [22], the separation-to-height ratio of the buildings in the streamwise direction, i.e. $\ell / h$ following the nomenclature introduced in Figure 1 , can be used to establish the following criteria: for $\ell / h<1$ a stable vortex is found [21]; as $\ell / h$ tends to 1 , higher speeds are found close to the ground. Note that the aforementioned behaviour is found when dealing with a uniform height over the domain. If significant differences between obstacle heights are found, the speed near the lower wall is significantly lower for the same separation-to-height ratio [21]. This last observation is supported by the work of Isyumov and Davenport [23], which shows that for large differences in height and a unitary separation-to-height ratio, the velocity near the lower wall is significantly smaller than what was predicted by Penwarden and Wise [22]. This discrepancy shows the importance of using three-dimensional approaches, since the various flow directions tend to behave independently.

Although the literature discusses certain observations regarding the flow features in these scenarios, there is a big need for more detailed information on the dynamical interaction of the flow structures in the various regimes. Furthermore, non-uniform street distributions and building heights will lead to a significantly more complex flow behavior, eventually responsible for the resulting distributions of pollutant concentration and temperature in urban flows. Those limitations suggest that understanding the actual behaviour of urban flows may require the implementation of more sophisticated approaches such as numerical simulations, specially in the study of the vortical structures.

Another important aspect of the study of urban flows deals with the characterisation of atmospheric boundary layer (ABL). This is the region of the atmosphere which is in contact with the surface of the Earth. Although the ABL effects are sometimes ignored in the study of urban flows, in more specific applications it is critical to have a good characterisation of this boundary layer. Hlevca and Degeratu [24], together with Counihan [25], described the basic characteristics of a neutral ABL, i.e. without thermal gradient. As with classic turbulent boundary layer (TBL), the ABL is formed by two layers with an additional overlapping layer that lies between those. In the lower layer, i.e. $20 \%-30 \%$ above the surface, the mean velocity exhibits a logarithmic behaviour [24] dependent on the roughness length, friction velocity and von Kármán constant. Note that the length roughness 
varies with the surface of the terrain under consideration and thus allows to particularise the law to the studied environment. However, Hlevca and Degenaru [24] explained that in the vast majority of engineering cases ABLs are modelled by means of a power law, thus approximating the ABL as a single layer. The power law is particularised to the application case by means of an exponent accounting for the terrain roughness.

Counihan [25] explains the limitations of ABL models observing that various anomalies are found to exist in the published literature. Those anomalies are found in the definition of the specific parameters in both the logarithmic and power laws. Discrepancies happen to increase as the terrain becomes rougher, which can be explained by the increase in turbulent structures and thus in flow complexity. As the flow becomes more complex, models tend to exhibit larger errors. The study of ABLs focuses on the development and particularisation of laws, i.e. on the selection of the coefficients representative of each case. However, these models tend to miss important information on turbulent structures. This explains why a part of the literature is focused on the study of turbulent processes within urban environments rather development of ABL models.

\section{Experimental studies}

The complex, three-dimensional flow around an obstacle was thoroughly investigated using flow visualization by Martinuzzi and Tropea [26]. Later, Cheng and Castro [27] conducted detailed measurements based on X-wire anemometry combined with laser-Doppler anemometry (LDA) of the flow in the roughness sublayer within a simplified urban environemnt. Their results confirmed the strong three-dimensional (3D) character of the dominant structures, as well as their interaction with the inertial sublayer. Further insight into the characteristics of the structures in the roughness sublayer (compared with those developing on smooth walls) was provided by Castro et al. [28] with additional measurements. These conclusions were complemented with spatial-correlation data obtained from particle-image velocimetry (PIV) by Reynolds and Castro [29]. On the other hand, the measurements by MacDonald et al. [30] focused on the characterization of turbulence statistics and the development of scaling laws for modelling purposes. Note that the considerations for adequate wind-tunnel scaling of the full-scale urban environment are discussed in detail by Wang et al. [31]. The effect of building geometry on the flow field and pressure distribution was assessed experimentally by Xing et al. [32].

A wide range of experimental studies have attempted to identify the most relevant flow features, in terms of interaction between obstacles or influence of the incoming flow direction [33,34]. Although in $\S 5$ we provide a more thorough assessment of flow structures in urban environments, here we highlight some important findings from the experimental perspective. For instance, the interaction between the incoming flow and the horseshoe vortex forming around an obstacle was documented experimentally by Nagib and Corke [35] through flow visualizations, and this phenomenon was later analyzed in detail through numerical simulation [36]. The importance of such structure knowledge for urban-boundary-layer parametrization was highlighted by Rotach et al. [37], whereas an attempt at a more explicit urban model was reported by Coceal and Belcher [38]. In this context, more realistic urban geometries were considered in the experiments by Rafailidis [39], while a shear-stress parametrization in a wind-tunnel experiment of a realistic urban geometry was proposed by Kastner-Klein and Rotach [40].

Detailed experiments of urban street canyons have been conducted in water channels with the aim of shedding light into the dominant dynamic processes [41,42]. Other wind-tunnel studies have focused on the relevant implications of scaling down relative to the full-scale models, and they have provided detailed assessments of the possible sources of discrepancy [43,44]. Takimoto et al. [45] went one step farther, by evaluating the implications of scaling down a full-scale model in the context of coherent structures in the flow. Another element present in large-scale models is the disparity of building height, an aspect which was assessed in detail in the work by Hagishima et al. [46]. Other elements present in full-scale models which are challenging to realize and measure in wind-tunnel experiments are the effects associated to temperature variation. Kovar-Panskus et al. [47,48] discuss 
the impact of these effects in two detailed experimental studies. Furthermore, the presence of trees may reduce pollutant concentration due to the fact that they disrupt the streams present in the city canyon, as thoroughly documented in a number of experimental studies [49-51]. Other elements, such as obstacles and obstructions in the streets, may have an important impact on the resulting flow, as discussed by Gayev and Savory [52]. Moreover, the potential of using modal decomposition to understand the role of the dominant flow structures was discussed by Kellnerova et al. [53], who performed proper orthogonal decomposition (POD) to shed some light into the data obtained with their PIV experiment. The role of modal decompositions in understanding the relevant flow structures in the context of urban flows will be discussed in $\S 5$ and in the Appendix. Next, we will discuss both open- and closed-environment experiments of urban flows.

\subsection{Open-environment experiments}

\subsubsection{Full-scale measurements}

Full-scale measurements are the main experimental strategy to study urban flows in an open-air environment. Urban flows are characterised by their complexity, which limits the applicability of controlled-environment approaches. Full-scale measurements allow to capture the natural complexity of urban flows and thus have the potential of providing an additional layer in the understanding of the physical phenomena involved in turbulent urban flows.

Fernando et al. [54] gathered the data obtained in the Mock Urban Setting Test (MUST) [55], an experiment designed to study the flow in an idealised neighborhood. The experimental site was conceived to mimic a uniformly distributed neighborhood but using idealised geometries so as to limit the complexity of the flow. The experimental site is composed by a $10 \times 12$ array of semi-trailer containers located in the Desert Test Centre of the U.S. Army Dugway Proving Ground. The experimental site was conceived taking into account the variation in wind direction. In fact, the site exhibits very frequently south-north winds which induce a skimming-flow regime within the test area. The instrumentation used in the MUST experiment varied with the study considered. Fernando et al. [54] used a tower with sonic anemometers as main measurement system. Additional anemometers were installed at the front of the obstacle array. The experiment was modelled in a wind tunnel in order to compare the obtained results. While the MUST experiment mainly focused on flow characterisation, other urban-flow studies deal with the thermal effects. As discussed in previous sections, thermal effects are critical in the energetic behaviour of an urban area. Thus, further experimentation is required to characterize such effects. Fernando et al. [54] gathered a series of experiments providing data on the urban heat island (UHI), which is characteristic of many cities in the world as a result of the nature of urban environments and anthropogenic activity. In fact, UHIs have a direct impact not only on meteorological phenomena but also on several different areas such as energy consumption, air pollution, agriculture etc. [56].

The Phoenix Urban Heat Island Experiment 2008 (PHEX-08) [57] gathered extensive temperature measurements aimed at validating UHI models. The PHEX-08 experiment analysed the circulation in street canyons due to temperature variation and how this circulation competes with the forced, i.e. vortex-induced, circulation that results from the interaction of the canyon fluid layer with the overlying ambient flow. The relation between both physical mechanisms, i.e. the balance between buoyancy and inertial effects, is described by means of the Richardson number. Temperature measurements were obtained by means of infrared (IR) thermography, sampling both the buildings in the street canyon and the surrounding areas. Furthermore, data was also obtained from a 300-m elevation. In the same line of work, other authors dealt with the UHI focusing on the underlying mechanisms and the parametrisation of the physical processes. Sharam et al. [56] explored the sensitivity of the UHI to urban parametrisations within the context of the Chicago metropolitan area (CMA) and its 
surroundings. A significant part of the available literature focuses on the development of physical parametrisations, i.e. models, that can help to identify the drivers of the UHI. Experiments tend to be used as verification or tuning methods for the developed models. In this case, Sharma et al. [56] gathered near-surface meteorological data from nine Mesowest stations located in the CMA to be compared with the simulations run during the study [56].

Moreover, Fernando et al. [54] presented the Hermoso Park dispersion study (HPS) which was carried out in Arizona to analyse the well-being of the residents in a $5-\mathrm{km}^{2}$ area. In this case the analysis focused on air quality and in particular the physical phenomena that affect it. The experiment covers measurements of meteorology, turbulence, $\mathrm{PM}_{10}$ and $\mathrm{PM}_{2.5}$ (i.e. particulate matter with diameter smaller than 10 and $2.5 \mu \mathrm{m}$ respectively) among others. However, Fernando et al. [54] cover exclusively the flow, turbulence and transport data. The measurement system mainly consists of a meteorological tower equipped with sonic anemometers to capture the aforementioned quantities. Moreover, balloons were launched at the same location to measure velocity, temperature and pressure as well as to gather PM data. Furthermore, additional meteorological stations were distributed over the site in order to gather additional measurements.

Vita et al. [58] present a complete study on the assessment of pedestrian distress in urban environments comparing full-scale experimental techniques with wind-tunnel approaches as well as numerical methodologies. They distributed eight sonic anemometers over the streets at a distance from the ground of $2 \mathrm{~m}$. In addition to those probes, a reference anemometer is permanently working. This reference probe was placed on the roof of a 62-m-height tower in a 10 meters mast with the purpose of reading the baseline conditions in the undisturbed zone. Both reference and testing probes were configured to record three-dimensional data. Note that the testing zone was selected to be highly gusted, precisely because the objective was to assess the different methodologies used in pedestrian distress studies. The full-scale testing was aimed at obtaining data on mean-flow speed. In addition, the data was also used to help characterising the flow. The measurements were then compared with the results of wind-tunnel experiments and numerical simulations.

\subsubsection{Reduced-scale measurements}

A natural variation of the full-scale experimental approach consists of testing a reduced model in an open environment. On the one hand, we know that urban turbulent flows exhibit a significant degree of complexity and thus are difficult to reproduce in a controlled environment. On the other hand, full-scale measurements tend to involve significant costs and their applicability is typically constrained to the study of the area where they are carried out. Reduced-scale open-environment experiments are an alternative way to approach the experimental study of urban flows. As far as the data-acquisition system is concerned, there is no significant difference, in principle, between the full and reduced-scale approaches. The data-acquisition strategy is specifically designed for the experiment in question.

Hirose et al. [59] presented a project to study wind-induced natural ventilation in cities and how it is affected by the surrounding flow. The experimental setup consisted of a 512 cubical blocks matrix where each block had a height of $1.5 \mathrm{~m}$. The total dimensions of the experimental site were $100 \times 50$ $\mathrm{m}^{2}$, and it was oriented such that the wind direction was most often aligned with the length of the site. The data-acquisition system was composed of 700 sonic anemometers equipped with a TR90-T probe. Those were installed at half-height distance from the top of the block. In addition, acrylic plates with pressure taps were also installed in the northwestern and southeastern faces of the blocks. Using the aforementioned setup two datasets were acquired. From the analytical perspective, the study rests on two major axes. On the one hand, the study of approaching flow conditions, characterised through the statistical descriptions of turbulent flows. On the other hand, the study revolved around the relation between pressure difference and velocity. The authors examined the probability distribution 
of the velocity at the horizontal wind direction by means of the streamwise velocity magnitude, the standard deviation as well as the velocity range in both southeastern and northwestern winds. As far as the relation between pressure and velocity is concerned, it was examined by means of the pressure coefficient, obtained for every position using the least-squares method.

\subsection{Closed-environment experiments}

Closed-environment experiments are, historically, one of the most widely used experimental approaches in fluid mechanics. As it was introduced in \$3.1, open-environment experiments gather the complexity of urban flows. However, it is precisely that complexity that limits the applicability range of such methods and thus the generality of the conclusions that can be extracted. It is at this point where controlled-environment experiments in wind tunnels can be helpful. In fact, by controlling the experiment, one can actually study the underlying properties of turbulence and its relation with exogenous parameters.

Wind-tunnel experiments rely on reproducing a given set of flow conditions in a confined space, i.e. the tunnel, in order to study a particular problem. In the case of urban turbulent flows, wind-tunnel experiments are used to study the fundamental mechanisms of turbulence as well as particular applications. Typically, one can distinguish experimental studies by their applicability range, i.e. whether they focus on the analysis of a general phenomenon or on the study of a particular application. In the case of urban flows, this distinction is visible in terms of flow behaviour and applications.

These measurements are usually employed as a verification method. It is very common to use several approaches, e.g. numerical, experimental, modelling, etc. to assess a given problem. Wind-tunnel testing involves inaccuracies inherent to the measurement methods and the confinement due to the size of the test section. Thus, it is very important to implement the necessary probe corrections $[60,61]$ for the measured quantities, as well as to account for aspects such as three-dimensional effects in the wind-tunnel test section [62]. Vita et al. [58] aim at reproducing the street-level test in full-scale open-environment with a scaled model such that it can be tested in the wind tunnel. Three different types of probes were considered: first, Irwin probes were used to determine the mean wind speed; although these are not the most accurate sensors for this type of measurement, their accuracy was considered to be sufficient their study. The main advantage lies on their omnidirectionality, which eases the installation process since no realignment is needed. Secondly, multi-hole probes such as Cobra probes, were used to measure the incoming wind speed on the top of the model. Multi-hole probes are typically used in high-resolution measurements of turbulent flows. However, those probes are limited by their insensitivity in flows slower than two meters per second as well as their directionality. The third measurement system was hot-wire anemometry, which overcomes the limitations of the two aforementioned system. Nevertheless, hot-wire anemometers exhibit limitations in terms of spatial resolution and sensitivity to wind direction [63]. To summarize, Irwin probes and hot-wire anemometers were used independently to measure the flow at pedestrian level, i.e. within the model. In addition, a Cobra probe was used to obtain data of the incoming wind speed. Similarly, the work by Gadilhe et al. [64] provides a clear assessment on the verification of measurements systems and models in urban turbulent flows. The work included a comparison of numerical predictions of wind flow with the data obtained from a wind-tunnel experiment. From the experimental point of view, a predictive model was developed to be able to compare both databases. Then a scaled model of the full-scale site was produced to be tested in the wind tunnel under suburban wind conditions.

Wind-tunnel experiments are also employed to study particular underlying effects of urban flows. For instance, Weerasuriya et al. [65] presented a study on the effect of twisted winds at a pedestrian level using a scaled model of the Tsuen Wan street in Hong Kong inside a boundary-layer 
wind-tunnel. The setup consisted of a series of wooden vanes twisted with a turning table such that the straight-streamlined flow was curved to obtain twisted wind conditions. The twist was set to obtain four distinct cases, i.e. $15^{\circ}$ and $30^{\circ}$ turning clockwise and counter-clockwise. The measurement system included a five-point probing system to obtain data of mean flow, turbulence intensities and yaw angles. At each point, the aforementioned quantities were measured at 12 discrete heights from $10 \mathrm{~mm}$ to $1000 \mathrm{~mm}$ for a sampling period of $65 \mathrm{~s}$ [65].

Other studies apply this kind of experiment to concrete applications. In the present work, we will focus on analysing two of the most recurrent applications, i.e. ABL and air-quality experiments. The study of ABLs using a wind tunnel typically requires modification of the pre-existing devices [24]. The main objective is to reproduce the conditions representative of an ABL such that wind-engineering applications can be further developed. Hlevca and Degeratu [24] designed a closed-circuit wind tunnel with a $7 \mathrm{~m} \times 5 \mathrm{~m} \times 3 \mathrm{~m}$ test section including slotted walls to reduce the blockage effect [24]. When dealing with turbulent-boundary-layer experiments, the distance between the inflow and the test section is critical. It is important to ensure that the boundary layer can properly develop before reaching the test section $[66,67]$. The authors face precisely that problem as the distance between the inflow and the first measurement point is only $3 \mathrm{~m}$, which was too short for the boundary layer to reach develop sufficiently [24]. Note that increasing the aforementioned distance is typically problematic as the dimensions of the wind tunnel tend to be constrained. Nevertheless, the authors addressed this limitation by installing a device consisting of a grid of horizontal bars with different squared cross sections installed at the entrance of the test chamber [24]. As far as the measurement system is concerned, unsteady velocity measurements were performed by means of a Dantec StreamLine hot-wire anemometer operated at constant temperature for three different wind velocities at three distinct locations in the lower half of the mid-longitudinal vertical plane of the experimental test chamber. Regarding the results, the authors presented a validation study as well as a complete analysis of the boundary-layer development.

Air-quality studies are a straightforward application of urban fluid mechanics. This area of study can be approached following different paths. On the one hand, one can focus on pollutants and more precisely on their dispersion. On the other hand, some studies approach this problem with a more general perspective, linking fundamental flow behaviour with air quality. In addition, there is a complete field of research dedicated to the study of pollutants from a chemical perspective. The present review focuses on the first two lines of work, leaving the chemical behaviour of pollutants for future works.

The pollutant concentration present in a given environment determines the air quality. From the experimental perspective, this type of study requires the modification of standard wind tunnels such that the pollutant can be properly introduced. Mo and Liu [68] used a water vapor source to introduce particles in the flow. Water vapor was generated with an atomiser to be released as a tracer from the line source [68]. The amount of released vapor-moisture mixture is controlled by the atomizer which is adjusted to match the needs of the different experiments. Note that the pollutant source needs to be properly designed such that the main flow is not significantly disturbed. Mo and Liu [68] actually estimated that the velocity of the flow induced by the line source is around 3\% - 6\% of the freestream velocity. By increasing the distance between the line source and the test section it would be possible to diminish the induced effects. However, increasing the distance between the source and the test area could modify the state of the particles reaching the test section. As far as the measurement system is concerned, it typically consists of the standard equipment with some additional sensors to measure the pollutant concentration. Mo and Liu [68] used a combination of hot-wire anemometry and humidity sensors to measure both the velocity fields and the vapor concentration. Vapour concentration is determined using a combination of two humidity and temperature sensors. Both temperature and 
humidity are measured at the test region and upstream of the water-vapor source in order to measure reference values. Using the gathered data, relative humidity is computed to be converted into vapor concentration, i.e. specific humidity. An alternative approach was considered by Talluru et al. [69], who released a tracer gas to simulate the contaminant, and measured the pollutant concentration through a photo-ionization detector (PID). In conclusion, one can see that considering pollutant dispersion within wind-tunnel experiment significantly increases the complexity of both the experimental process and the obtained data. In addition, extensive verification has to applied to ensure that the observed phenomena are indeed representative of the physics under study.

Another approach to the study of air quality in urban environments consists of analyzing the fundamental behaviour of the flow. Instead of focusing on the actual drivers of air quality, e.g. pollutant dispersion, the idea is to focus on flow behaviour, which is then characterized using a model. Hertwig et al. [70] employed the aforementioned approach to study the relation between meteorological variability and air quality in urban environments. They combined open-environment observations with wind-tunnel experiments to compare with the prediction of several models [70]. A $20 \mathrm{~m} \times 3.5 \mathrm{~m} \times 1.5 \mathrm{~m}$ open-return boundary-layer wind-tunnel was used to test a scaled model of a specific area in south London. The full-scale domain covers $700 \mathrm{~m}$ of mixed residential and commercial neighborhood [70]. Measurements were taken using a two-component laser-Doppler anemometry system. The aforementioned approach provides relevant results regarding the relation between flow behaviour and air quality. However, air quality is introduced with modelling instead of testing in the wind tunnel.

\subsubsection{Closed-environment experiments with particle image velocimetry}

Particle image velocimetry (PIV) is an experimental method to study a certain region within a turbulent flow. Essentially, reflective particles, i.e. seeding, are introduced in the flow and their trajectories are traced. The position of the particles is measured using a camera system which detects them. Note that the seeding has to be properly adjusted, i.e. a sufficient amount of particles have to be introduced without having a significant impact on the flow behaviour. On the other hand, a scaled model of the geometry needs to be produced. Once again, the model needs to be properly set to mimic the flow behavior around the obstacles under study. The development of the model is typically simple in the case of urban environments since the obstacle array consists mainly of cubic shapes. However, the size of the urban canopies is usually a limiting factor for such experiments. Nevertheless, the PIV technique, when properly implemented, is a very powerful tool to study complex flows.

Monnier et al. [71] presented a research-oriented study that aimed to test the applicability of PIV methodologies in the investigation of flows over an urban-like obstacle array. The authors relied on work of Nagib et al. [72] to design the experimental strategy. The experiment mainly consists of PIV measurements varying the incidence angle of the incoming freestream flow in a scaled urban environment. The measurement system introduced in the wind tunnel consists of an array of three hot wires mounted over a vertical traverse system enabling measurement of the velocity profiles starting from a position close to the lower wall and extending approximately $400 \mathrm{~mm}$ above it [71]. As far as seeding is concerned, an atomiser is used to control the size of the particles. Seeding is injected by three inlets at the floor of the facility. Using PIV methodology, the authors verified the correlation between obstacle spacing and flow regimes presented by Oke [15]. Further analysis also brought significant results in terms of energy exchange between the different boundary-layer regions. In conclusion, the study presented by Monnier et al. [71] shows how the PIV strategy can produce relevant results that deepen the understanding of the fundamental drivers of urban flows.

Ferreira and Ganapathisubramani [73] examined the application of the PIV methodology to the estimation of the pressure distribution in an urban canopy. The authors compared the pressure field derived from in-plane velocity PIV measurements with numerical-simulation data in order to assess 

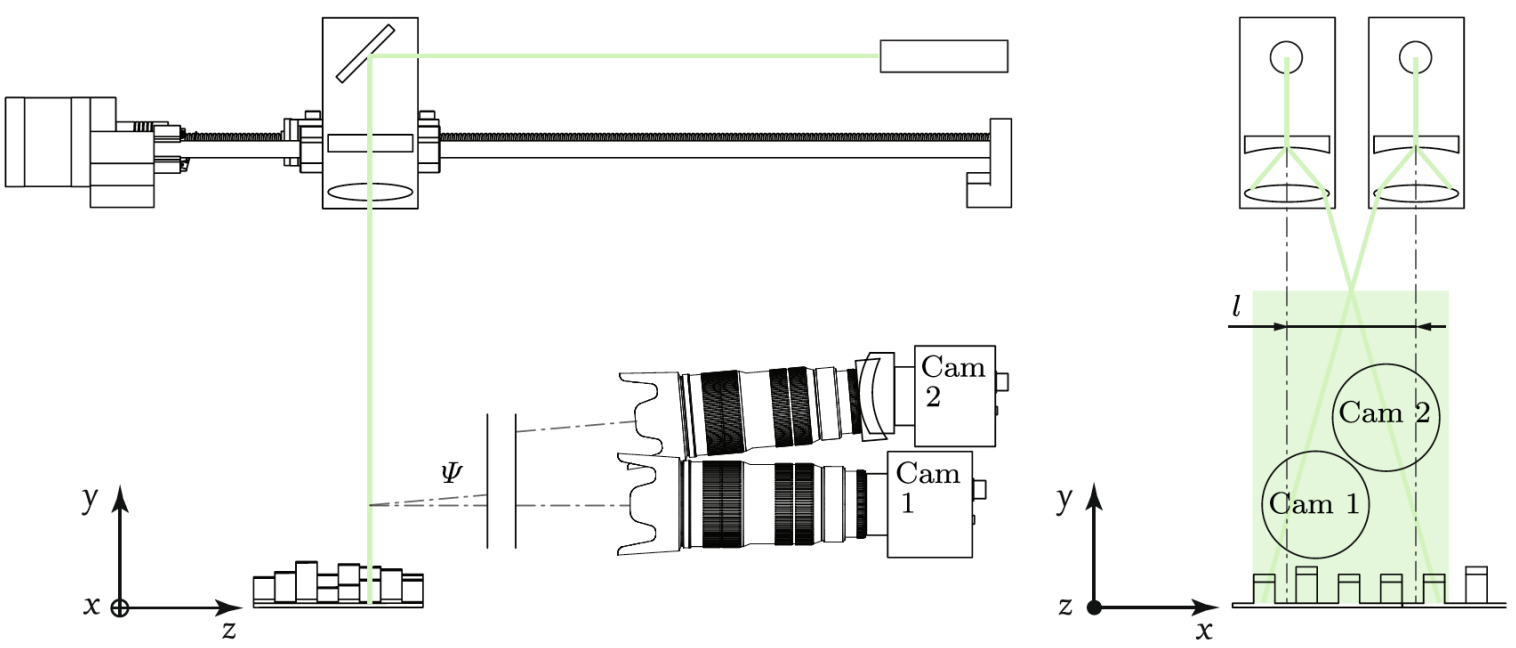

Figure 4. (Left) Front and (right) side view of PIV setup and measurement system used in the uniformand variable-height experiments by from Ferreira and Ganapathisubramani [73]. Reprinted from Ref. [73], with permission of the publisher (Springer Nature).

the application of PIV measurements to pressure estimations. The geometrical model consisted of two arrays of obstacles, one with uniform height and another one with variable height. The measurements in each of the arrays are obtained using a planar PIV in the streamwise- wall-normal plane [73]. Lasers were installed over the test section to direct the beam towards a set of optics that allowed to redirect the light sheets to predetermined locations. Cameras were mounted on two-dimensional linear translation stages. One of the cameras was tilted to focus on particular planes [73]. The whole system is mounted such that the canopy is fully illuminated. Figure 4 shows a schematic representation of the aforementioned PIV setup.

As far as the estimation of pressure fields is concerned, the authors use the velocity fields obtained from PIV measurements to run two numerical simulations. Ferreira and Ganapathisubramani [73] also assess the uncertainty of the methodology, with focus on the velocity fields. The main source of error comes from the use of a planar PIV. We know that urban flows are complex and three-dimensional. In this way, the use of a planar measurement system will inevitably introduce errors. The authors used numerical data to evaluate the magnitude of the error, concluding that the uncertainty remained constrained. Furthermore, Ferreira and Ganapathisubramani [73] analysed the error of the numerical methods employed to obtain the pressure estimation. In conclusion, the methodology, despite the uncertainty, is adequately validated [73]. The study in question shows how the PIV methodology can actually be combined with numerical simulations to obtain specific magnitudes of the flow.

\subsection{Experiments combining various techniques}

The work by Vita et al. [58] was aimed at comparing open-environment and wind-tunnel experiments within the context of urban flows. The authors measured mean-flow velocity as well as the corresponding standard deviation. The mean wind-speed data exhibited a good qualitative agreement with the wind-tunnel data, both having the same trend over the measurement positions. On the other hand, the wind-tunnel data was within the standard-deviation range found in the full-scale measurements at several measurement locations. Vita et al. [58] also compared the results with numerical simulations and concluded that the wind-tunnel exhibited deviations with respect to the full-scale results particularly in the recirculation region. Through analysis of their numerical data, Vita et al. [58] found that the velocity streamlines had a rather complex behavior. In conclusion, the work presented by Vita et al. [58] provides a clear picture on the suitability of the methods available 

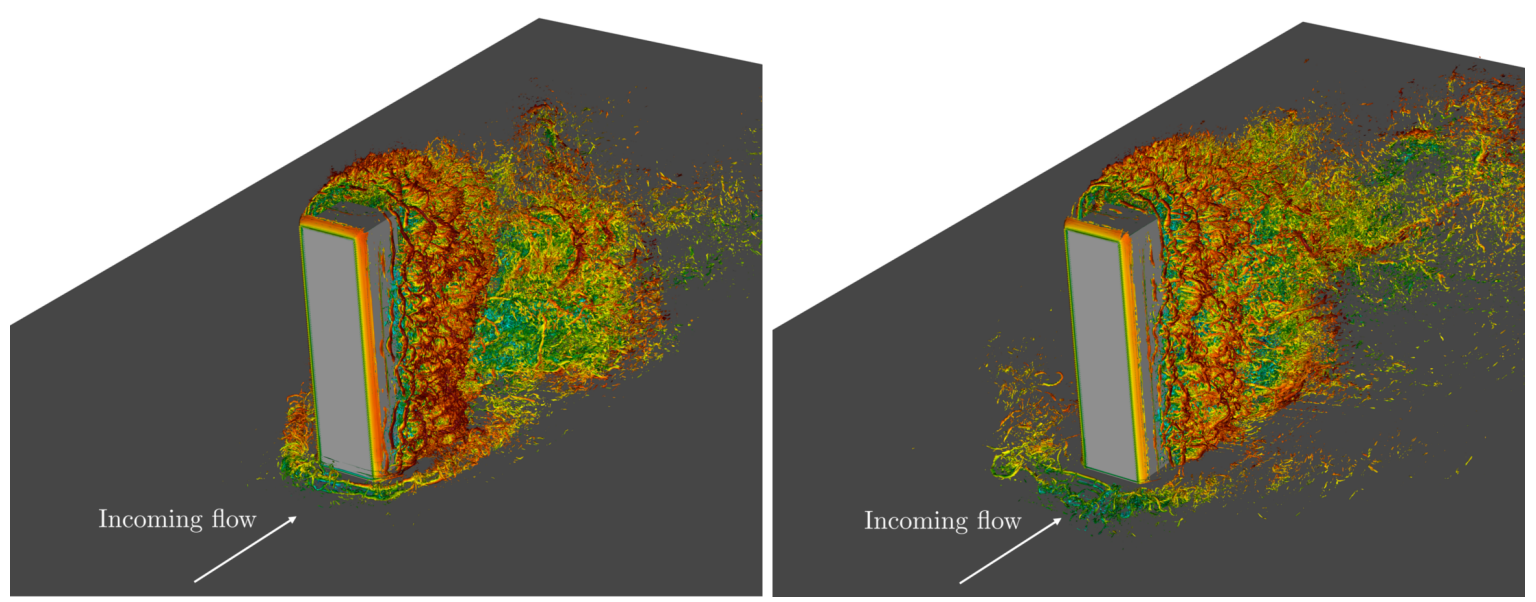

Figure 5. Instantaneous flow visualizations showing vortical structures [18] around a wall-mounted square cylinder, with (left) turbulent- and (right) laminar-inflow conditions. We show $\lambda_{2}$ isosurfaces of -40 (based on $U_{\infty}$ and $w_{b}$ ), and the structures are colored by streamwise velocity, ranging from (dark blue) -1.2 to (dark red) 1.8. Figures produced from the databse by Vinuesa et al. [36].

to study urban flows. The main conclusion presented by the authors suggests the integration of a multi-method approach to deepen the understanding of urban flows.

\section{Numerical studies}

In Figure 5 we show the instantaneous flow around a wall-mounted obstacle [36] for two cases, i.e. with a laminar and a turbulent inflow, which was obtained with a highly resolved simulation method called direct numerical simulation (DNS). In this high-fidelity simulation approach, the governing equations of the flow (i.e. the Navier-Stokes equations) are discretized and solved numerically in a mesh fine enough to capture the smallest turbulent structures. The multi-scale character of the turbulent flow present in urban environments can be observed in Figure 5, where the vortical structures within the wake exhibit a significant range of sizes and energetic content. This high complexity requires the use of appropriate meshes and numerical methods in order to avoid distorting the underlying physics with numerical artifacts. When properly designed high-fidelity simulations are employed, it is possible to obtain a representation of the flow field with the highest level of detail, well beyond what can be achieved with currently available industrial simulations based on Reynolds-averaged Navier-Stokes (RANS) models (where no turbulent scales are simulated) or coarsely resolved large-eddy simulations (LES, where only the largest turbulent scales are resolved). Although the flow exhibits certain similarities in Figures 5 (left) and (right) due to the fact that the sharp cylinder edges prescribe the separation location, their different inflow conditions lead to a number of visible particularities in each case. The recirculation region on the leeward side of the obstacle is shorter in the turbulent-inflow case, and interestingly the horseshoe vortex forming around the cylinder is much more prominent in the laminar-inflow configuration. This is due to the fact that, as discussed by Vinuesa et al. [36], the incoming turbulence modulates the horseshoe vortex in Figure 5 (left), thus affecting its formation process. Furthermore, as can also be observed in Figure 5, the wake is wider in the turbulent-inflow case close to the obstacle, whereas the laminar-inflow configuration exhibits a wider wake farther downstream.

A number of studies have investigated the flow in urban environments through numerical simulations $[16,74,75]$, and in most cases the results are validated with wind-tunnel experiments. These numerical studies have documented relevant effects in urban flows which should be studied in even more detail. It is interesting to differentiate the articles simulating urban flows that simply focus on characterizing the flow in urban environments $[76,77]$ (in some cases even using more realistic representations of the shape and distribution of the buildings following the information contained in 
real planes of specific cities), from the articles considering more realistic situations, although generally using simpler representations of the urban environment (i.e. arrays of regular obstacles). In the latter case, it is possible to identify (among others) some studies comparing several wind-comfort criteria [78], using stratification for calculating surface cooling rates [79] or studying the concentration levels and dispersion of a pollutant release [80].

The characterization of the flow in realistic urban environments is mainly motivated by studying the influence on small-scale fluctuations of atmospheric flow. The origin of such turbulence fluctuations could be produced (among other factors) by the presence of high-rise buildings, which in some cases produce gusty winds. Numerical weather prediction models provide a general understanding of the wind system in urban flows, however these tools cannot properly reproduce wind fluctuations, thus requiring more sophisticated models, such as LES, combined with different strategies. Generally, LES is able to characterize the flow in the urban-roughness sublayer, which is the atmospheric layer from the ground to $2-5$ times the average heigh of the building array. This layer is essential to predict the pollutant dispersion, air quality and weather conditions. Regarding the several techniques used to characterize the flow, it is interesting to mention the following works. Nakayama et al. $[81,82]$ performed LES using the Smagorinsky model [83] for the sub-grid scales to simulate a strong wind event in Tokio using a real meteorological setting performing mesoscale simulations. These authors extended the technique by Kataoka and Mizuno [84] to combine turbulent flows from numerical weather-prediction simulations with computational-fluid-dynamics (CFD) models. Giometto et al. [85] performed LES with two closure models for the sub-grid scales, the Smagorinsky model combined with a wall-damping function as in Ref. [86] and the scale-dependent model with Lagrangian averaging of the coefficient by Bou-Zeid et al. [87]. These authors combined LES with immersed boundary conditions, representing the buildings by a discrete-forcing immersed-boundary method. The authors show that this formulation is valid to model the turbulence and dispersion in a real urban roughness sublayer, in particular representing the urban city of Basel (Switzerland). Inagaki et al. [88] studied the validity of the outer-layer scaling for turbulence statistics and coherent structures under neutral stratification combining LES with a Smagorinsky model with the lattice-Boltzman method. The authors used a realistic urban geometry modelling the city of Tokio (Japan). They found energetically predominant structures throughout the entire boundary layer, probably driving the flow dynamics, but this information was insufficient to identify the leading mechanism, i.e. whether it was associated to the inner or the outer layer. Finally, Crylls et al. [89] introduced a new method using a LES approach (with periodic boundary conditions) to model the statistically-steady state of non-neutral urban boundary layers. This method freezes transient simulations into a steady state, showing a good performance in both stable and convective conditions.

Regarding the evolution of pollutant dispersion, the review of Belcher [90] reflects the importance of distinguishing between the characteristics of the concentration distribution in the near and far fields, as shown by Theurer et al. [91]. Close to the source, the dispersion depends on the source position and the shape and distribution of the buildings, while in the far field, a Gaussian plume model is sufficiently accurate to model the dispersion (see more details in Refs. [92,93]). Belcher [90] identified three main dispersion processes in the urban environment: (i) the contribution of the shear layers found at the rooftop in the interaction of the flow above and within the canopy; (ii) the topological dispersion, dividing streamlines when the flow impinges on buildings; and (iii) the presence of secondary sources behind the building wakes. Motivated by the lack of information regarding the effect of wind direction and the three-dimensional structure of the dispersion plume in urban areas, Coceal et al. [94,95] performed DNS to compute the statistics of turbulent flows in arrays of obstacles, and Brandford et al. [96] extended that work, also using DNS, studying the evolution of a point-source release of a passive scalar in a urban environment modelled by an array of regular obstacles (cubes). These authors studied the variation of the mean concentration profiles in three different cases, where the angle of the flow direction and the position and number of the point sources was different (three different pressure-gradient directions). They identified six main processes affecting the plume: (i) skewing, (ii) 
meandering, (iii) detriment by turbulent dispersion, (iv) entrainment and scalar release producing secondary sources, (v) scalar advection downstream and (vi) lateral and topological dispersion due to turbulent fluctuations. To increase the insight into pollution-dispersion mechanisms, Michioka et al. [74] performed LES using dynamic subgrids-scale (SGS) models for the unresolved scales [97,98] to simulate the flow over an array of obstacles with different aspect ratios, showing that the ratio of the turbulent to the advective pollutant flux was almost constant in all the cases, although the turbulent field was the main contributor to the street-canyon ventilation.

The aforementioned work [74,94-96] assumed that turbulent boundary layer was fully developed over large urban areas, with uniform properties. However, more realistic numerical studies take into account that the boundary layer has to adapt to the changing surface roughness, and only a few studies resolve explicitly the roughness transition to simulate the flow $[99,100]$. Following this research direction, Tomas et al. [101] performed LES in a simplified geometry consisting of over 20 streets. They employed the eddy-viscosity model by Vreman [102] to account for the unresolved turbulent stresses. These authors investigate on a rural-to-urban roughness transition the effect of stable stratification on pollutant dispersion, using two lines sources of pollutant. Their results showed the relevance of the vertical momentum fluxes due to turbulence for dispersion of scalars in urban flows. More specifically, they showed that due to the slower internal boundary-layer growth in stable conditions, concentration remains 17\% larger. This can be observed in more detail in Figure 6, which shows instantaneous visualizations in the neutral- and stable-stratification cases. Moreover, the advective pollutant flux is still relevant up to a long distance downstream of the rural-to-urban roughness transition. This work was extended by Tomas et al. [103], considering various urban canopies with obstacles using different aspect ratios. These authors studied in depth the influence of such aspect ratio in the pollutant dispersion, surface forces, velocity statistics and boundary-layer depth, trying to identify the dominant mechanisms of pollutant removal from street canyons and their evolution in the transition region. Using a similar eddy-viscosity-based LES approach, Eisma et al. [104] extended the previous work [103], with the aim of assessing the impact of a sound barrier on the urban region. This type of sound barrier is used to reduce the sound levels from highways on neighbouring cities, and the results by Eisma et al. [104] suggest that they may have a significant impact on the pollutant concentration and air quality in the urban area. More specifically, the presence of the plane fence produces a deeper internal boundary later. It is also shown that the mean concentration fields scale with the internal boundary-layer bulk velocity and depth. These results are different from the work by Vinçont et al. [105], who studied the evolution of the passive scalar in the wake of a two-dimensional obstacle, finding the highest concentration in the wake when the line source was located at the wall downstream the obstacle, in contrast to the previous work [104], where the sound barrier was located downstream of the source.

Motivated by the need of performing accurate simulations with a reduced computational cost, several authors have developed urban-dispersion models, which are suitable for air-quality and emergency-response applications, although the continuous search of novel approaches that are able to model complex turbulent flow processes is still a research topic of high interest. For instance, Soulhac [106] developed the governing equations which formed the origin of a new operational dispersion model, SIRANE [107]. Using an array of cubes, Hamlyn et al. [108] developed an accurate and very simple model for dispersion, in good agreement with experimental results. Belcher [90] and Belcher et al. [109] showed the explicit dependence of the concentration on the flow parameters and the geometry by the development of a simple analytical model of passive scalar for a regular street network. These authors found that the near-field regime was leading the flow, were the vertical detainment out of the street network was influencing the flow above. Finally, Goulart et al. [110] created a model to predict mean concentration which was validated with DNS results. The DNS modeled the dispersion of a passive scalar from a continuous source release. The authors identified three regions as function of the movement of the vertical fluxes: the near-field, characterized by vertical detainment; the transition region, characterized by vertical detainment and re-entrainment; and the 
(a)

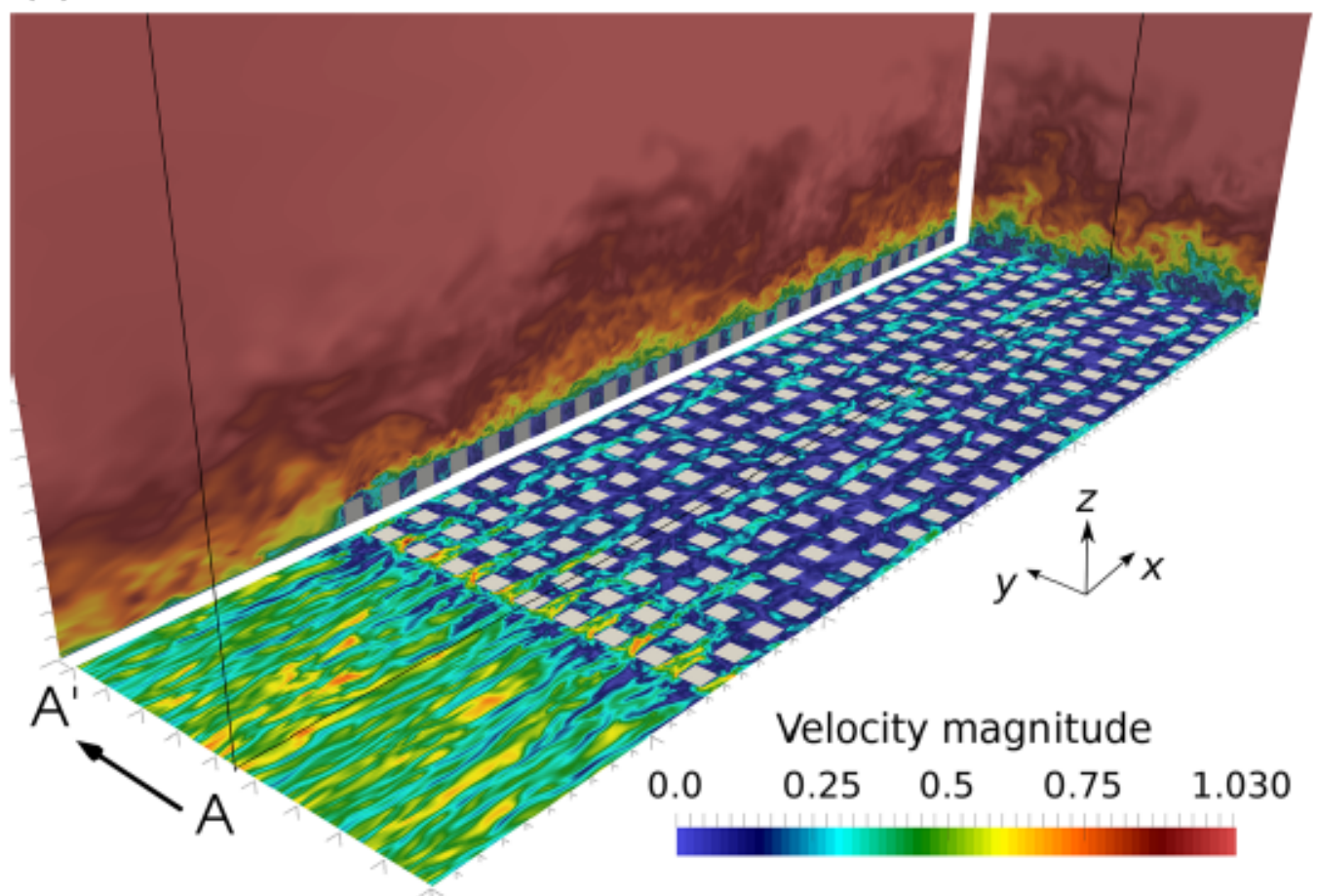

(b)

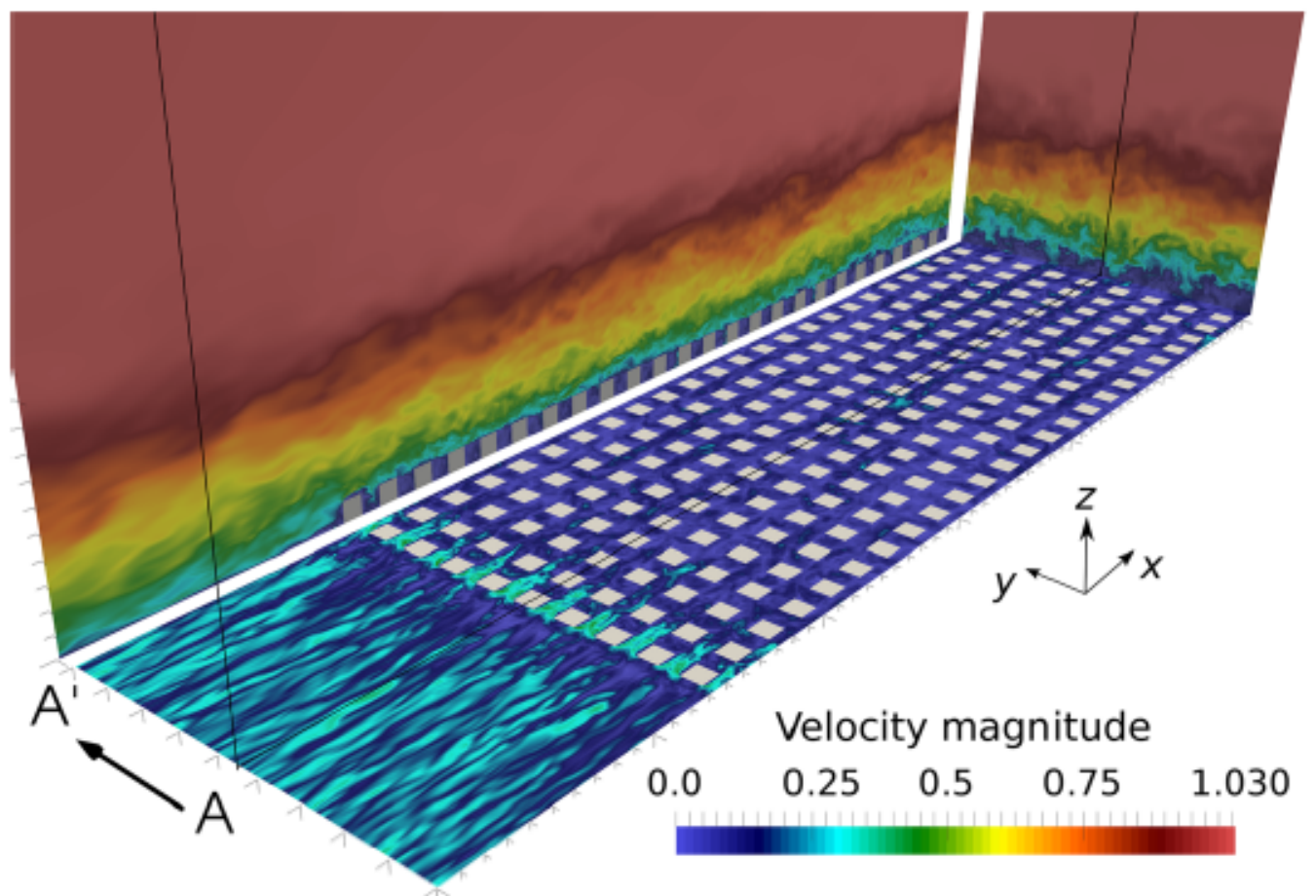

Figure 6. Instantaneous visualization showing the velocity magnitude in a rural-to-urban transition case. (Top) Neutral- and (bottom) stable-stratification cases are shown, with the velocity magnitude normalized by $U_{\infty}$. The centerplane is projected on plane $\mathrm{A}^{\prime}$, and the horizontal plane is located at $z / h=0.1$. Reprinted from Ref. [101], with permission of the publisher (Springer Nature). 
far field, characterized by similar quantities of vertical detainment and re-entrainment. Based on the DNS results, the model uses a formulation based on volume-averaging the governing equations and includes the flow characterization by horizontal advection and vertical detainment and re-entrainment.

\subsection{Wall models}

Another important aspect of urban-flow simulations is the Reynolds number: a non-dimensional parameter representing the ratio of inertial and viscous effects in the flow, which is proportional to the flow speed and the size of the problem, and inversely proportional to the fluid kinematic viscosity. Typical Reynolds numbers in atmospheric boundary layers (ABLs) are several orders of magnitude larger than those of most urban-flow studies based on turbulence models or wind-tunnel experiments [111]. The computational cost of LES and DNS roughly scales with the square and the cube of the Reynolds number respectively [112], which implies that these simulation approaches are prohibitively expensive for full-scale urban-flow simulations. The mean flow in the so-called inertial sublayer has been widely studied in the ABL community, and it is known that (in neutral conditions) it can be described in terms of a logarithmic law [13]. This law includes two parameters representing the surface-roughness and the surface-displacement lengths, which are both characteristic of the urban canopy under study. The logarithmic description of the inertial sublayer led to the idea of using the so-called wall models, which in principle would allow to replace the whole region very close to the wall by a model defining a surface shear stress matching such logarithmic behavior. This is the cornerstone of most atmospheric models, which avoid resolving the highly computationally expensive scales close to the wall. This wall-model approach, combined with an eddy-viscosity-based LES, was used by Cheng and Porté-Agel [100] to study a simplified urban model at high-Reynolds-number conditions. They defined the shear-stress boundary condition based on the Monin-Obukhov similarity theory [113], and their results are very relevant for the improvement of currently existing urban-canopy prediction models. A similar wall-modeled LES was adopted by Giometto et al. [114] to study a real urban geometry in the city of Basel (Switzerland) at high Reynolds number, for which field measurements are available [115]. In their study they adopted a slightly different approach, considering the LES model by Bou-Zeid et al. [116] and the Moeng model [117] for the wall boundary condition. The analysis by Giometto et al. [114] identified turbulent kinetic energy (TKE) production mechanisms above the average building height, as well as the corresponding transport phenomena towards the lower street levels.

\section{Flow structures}

Coherent flow structures play a critical role in urban environments, since they are responsible for the mechanisms leading to the wind patterns and gusts, thermal distributions and pollutant-concentration fields. Although these structures have been identified and documented in experimental studies, numerical simulations enable sufficient spatial and temporal resolution to evaluate their effect in the dynamic processes taking place in an urban environment. As discussed in $\S 4$, Vinuesa et al. [36] compared the coherent structures resulting in the flow around a wall-mounted obstacle when the incoming boundary layer was laminar or turbulent, using DNS. As observed in Figure 5, the sharp edges of the obstacle fixes the separation location regardless of the inflow conditions. However, the resulting structures and the topology of the wake significantly differ in both cases. In particular, Vinuesa et al. [36] identified a complex modulation mechanism [118] in the turbulent-inflow case from the incoming boundary layer on the horseshoe vortex forming around the obstacle. This highlights the importance of having well-defined inflow conditions, first for flow reproducibility, and second to ensure an adequate characterization of these types of complex phenomena. It is also important to highlight the need of employing an adequate resolution in the simulations, which may allow to completely characterize elusive phenomena, such as the interaction of the incoming atmospheric boundary layer with an ice sheet [119], a physical process with very important implications 


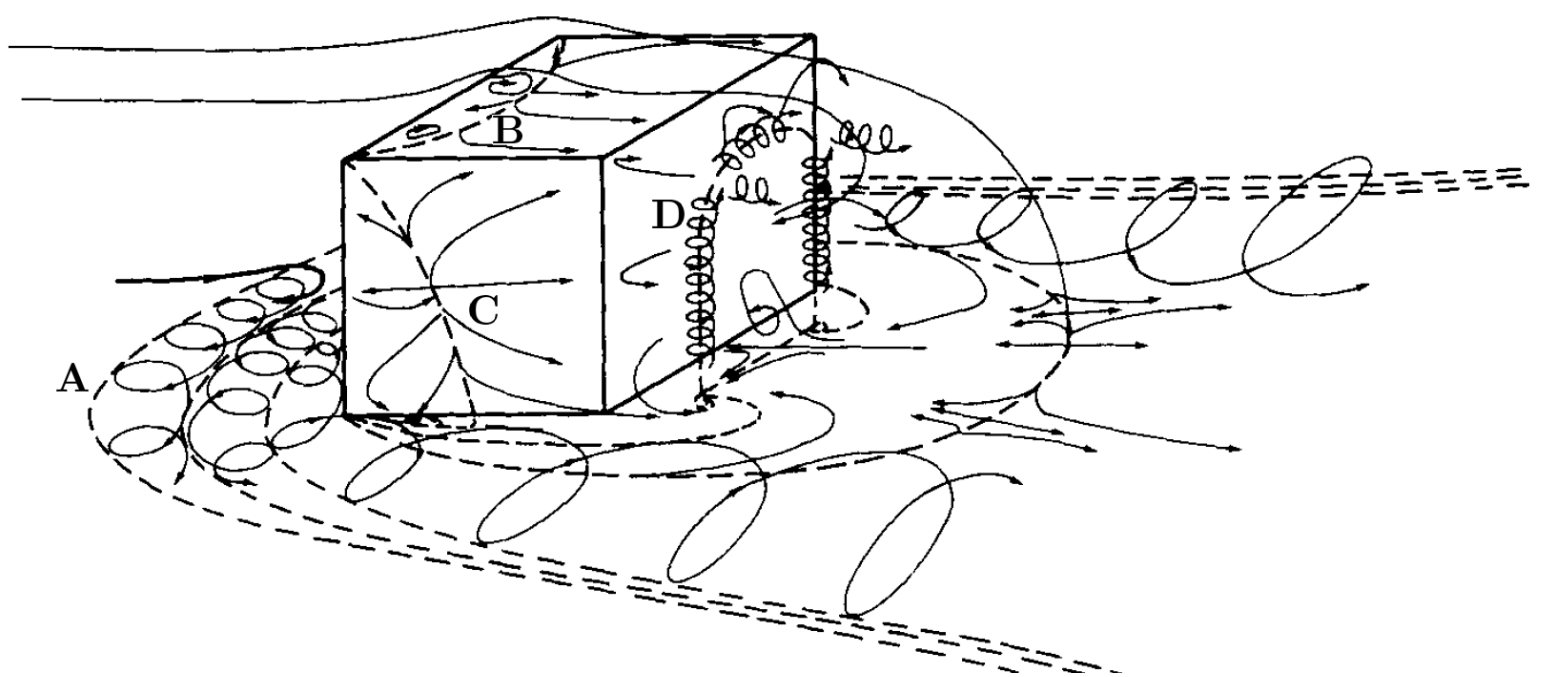

Figure 7. Illustration of the main vortical structures around a surface-mounted obstacle. Adapted from Ref. [121], with permission of the publisher (Cambridge University Press).

in the interpretation of climate models, since it affects the large-scale atmospheric circulation on continental scale [120].

Returning to urban flows, Hunt et al. [121] conducted one of the first studies aimed at identifying the structures around a wall-mounted obstacle, using flow visualization. In Figure 7 we show the schematic representation proposed by Hunt et al. [121], where a total of 4 vortical structures are identified: the horseshoe vortex (A) which forms around the obstacle; the roof vortex (B) and the vortices on the obstacle sides (C), which have a close interaction with the wake; and finally the well-known arch vortex (D) on the leeward side of the obstacle, which will be discussed in detail below. Note that there are a number of methods used to identify coherent structures, and although they do not exhibit significant differences when applied to turbulent flows [122], they may emphasize slightly different phenomena when applied to urban environments [71]. Furthermore, we argue that the use of adequate thresholds may help to discern intense event inherent to turbulent fluctuations and large-scale effects induced by the geometry. A more recent study by Sousa [123] provides a detailed picture of the various structures around a wall-mounted cube using PIV. Sousa [123] used (among others) the so-called swirling strength [124] to obtain time-averaged structures. Note that the swirling strength $\lambda_{c i}^{2}$ is essentially the square of the imaginary part of the eigenvalues from the velocity-gradient tensor.

Further insight into the characteristics of these vortical structures was provided by Becker $e t$ al. [125], who considered a series of wall-mounted obstacles with aspect ratios $b / h$ varying from 0.28 to 4 . They employed LDA and oil-film visualizations in wind-tunnel experiments where particular care was taken to ensure inflow conditions representative of urban environments. In Figure 8 we show their oil-film visualizations for an obstacle with $b / h=2$ and $w_{b} / h=0.29$; in this figure, the AOI is progressively increased from $15^{\circ}$ to $60^{\circ}$. These results indicate the evolution of the arch vortex, which was extensively characterized by Martinuzzi [126] for AOI $=0^{\circ}$, for increasing incidence angle. The first observation is the dislocation of one of the legs of the vortex, which progressively rotates until reaching the side of the obstacle for AOI $=45^{\circ}$. Interestingly, an additional increase of AOI to $60^{\circ}$ results in a displacement of the vortex leg to the top of the obstacle, a fact that significantly affects the momentum-transfer mechanisms within an urban canyon. Note that other studies assessing the characteristics of the arch vortex include Refs. [127,128].

As discussed by Monnier et al. [71], there is a wide range of criteria to identify vortical structures, and perhaps the most straighforward approach is to evaluate the vorticity components. In their study, they considered a wind-tunnel test emulating the geometry of the Mock Urban Setting Test (MUST) experiment, which was conducted at the Dugway Proving Ground in Utah as documented 

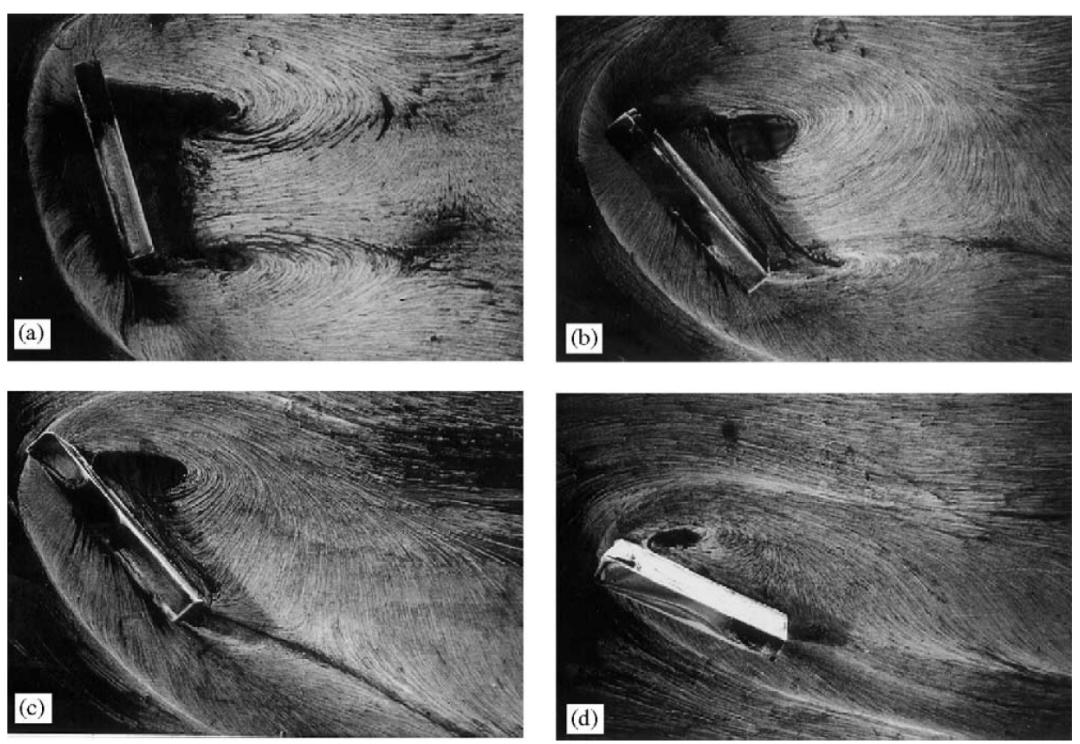

Figure 8. Oil-film visualizations of the vortical structures around a wall-mounted obstacle with $b / h=2$ and $w_{b} / h=0.29$. The following incidence angles are shown: (a) $\mathrm{AOI}=15^{\circ}$, (b) $\mathrm{AOI}=30^{\circ}$, (c) $\mathrm{AOI}=45^{\circ}$ and $(\mathrm{d}) \mathrm{AOI}=60^{\circ}$. Reprinted from Ref. [125], with permission of the publisher (Elsevier).

by Biltoft [55]. In this simplified urban environment, Monnier et al. [71] considered a set of buildings with $b / h=5$ and $w_{b} / h=1$, arranged into four rows and three columns. Regarding the building arrangement, they used $s / h=2$ and they considered two values of $\ell / h$, i.e. 1.5 and 4 . By visualizing the wall-normal and spawnise mean vorticities, $\Omega_{z}$ and $\Omega_{y}$ respectively, they could also identify the arch vortex and assess the effect of AOI. Perhaps the most relevant contribution of this visualization is the fact that it highlights the relevance of using a threshold to identify coherent structures. In this case, they considered the modulus of the spatially-averaged vorticity vector $\Omega=\sqrt{\Omega_{x}^{2}+\Omega_{y}^{2}+\Omega_{z}^{2}}$ as a threshold, which allows to clearly see the rotation of the arch vortex when changing AOI. Note that using local thresholds (as opposed to global) can be quite important in order to adequately identify coherent structures, so as to take into account the non-homogeneity of the flow induced by the walls (as it is common practice in wall-bounded turbulence [129-132]).

Another popular method for vortex identification was proposed by Hunt et al. [133] and it is commonly known as the $Q$ criterion. This method is based on the second invariant of the velocity-gradient tensor $Q=-1 / 2\left(S^{2}+R^{2}\right)$, where $S$ and $R$ are the corresponding symmetric and antisymmetric parts of the tensor (also known as the strain-rate and rotation tensors, respectively). The $Q$ criterion was used by Krajnovic and Davidson [134] to identify the vortical structures around a wall-mounted cube; note that this technique has the advantage of correctly identifying vortices even under high shear. Another popular method for vortex identification is the so-called $\lambda_{2}$ criterion by Jeong and Hussain [18], which is based on analyzing the eigenvalues of $S^{2}+R^{2}$. In particular, they define a vortex core as the connected area where $S^{2}+R^{2}$ has two negative eigenvalues. If the eigenvalues are defined as $\lambda_{1} \geq \lambda_{2} \geq \lambda_{3}$, the vortex core can be identified as the region where $\lambda_{2}<0$. There are other vortex-identification methods based on the velocity gradient, such as the $\Delta$ criterion by Chong et al. [135] or the approach by Kida and Miura [136] based on regions of low pressure combined with a swirl condition.

Besides the previously mentioned methods, which are based on the velocity gradient, there is another approach which is based on an integral quantity instead: the normalized angular momentum $\Gamma_{1}$ proposed by Graftieaux et al. [137], which is defined as follows:

$$
\Gamma_{1}\left(\mathbf{x}_{p}\right)=\frac{1}{D} \int_{D} \frac{\left(\mathbf{x}-\mathbf{x}_{p}\right) \times \mathbf{u}(\mathbf{x})}{\left|\mathbf{x}-\mathbf{x}_{p}\right||\mathbf{u}(\mathbf{x})|} \mathrm{d} \mathbf{x},
$$



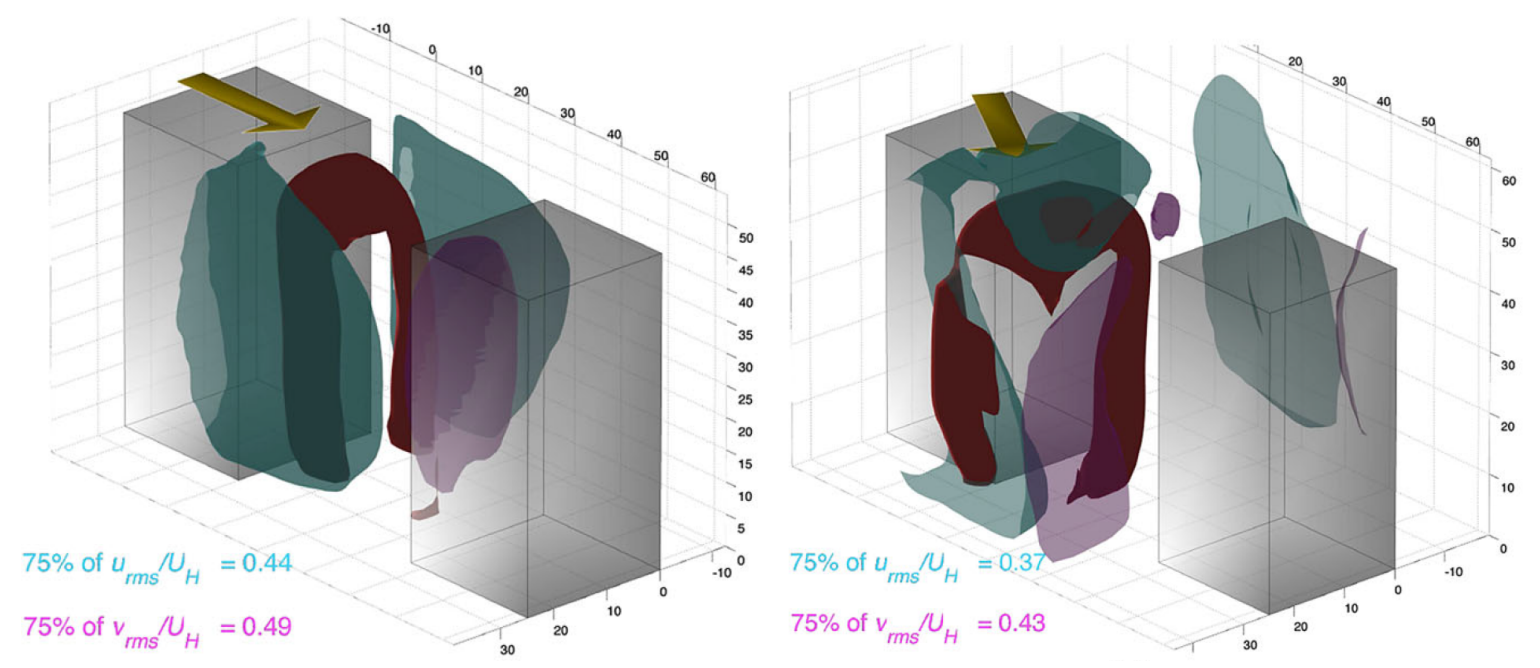

Figure 9. (Left) Isosurfaces of (red) $\Gamma_{1}=0.4$, (blue) streamwise and (pruple) spanwise root-mean-squared (rms) fluctuations, showing thresholds equal to $75 \%$ of the value indicated on each panel, for AOI $=0^{\circ}$. (Right) Same as (left), with $\Gamma_{1}=0.35$ and AOI $=30^{\circ}$. Reprinted from Ref. [138], with permission of the publisher (Springer Nature).

where $D$ is a small integration domain centered around point $\mathbf{x}_{p}, \mathbf{u}(\mathbf{x})$ is the velocity vector, $\times$ denotes vector product and $|\cdot|$ is the vector modulus. This method was used by Monnier et al. [138] to identify the various relevant regions in the flow between two obstacles, as can be observed in Figure 9. On the leeward side of the upstream obstacle the flow exhibits the arch vortex, with the two legs having flow rotating in the wall-normal direction and the roof exhibiting flow rotating in the spanwise direction. Note that the modulus of the normalized angular momentum $\Gamma_{1}$ is used to define the visualized isosurfaces. The windward side of the downstream obstacle exhibits a region of high spanwise turbulence intensity, and this system is flanked by two regions of high streamwise fluctuations on both sides of the obstacles. Although this flow is observed for an AOI of the incoming $\mathrm{ABL}$ of $0^{\circ}$, see Figure 9 (left), the various elements become reoriented for progressively higher AOI, as observed in Figure 9 (right). This reorientation was also documented in a simpler setting by Rempfer et al. [139]. Note that this particular urban geometry was designed to emulate the flow behavior in a downtown area, with relatively high buildings compared to the street width, and therefore it exhibits skimming-flow regime [16]. One approach to obtain deeper insight into the flow is by analyzing instantaneous three-dimensional fields, and identifying the coherent structures responsible for the observed behavior [129-132]. Furthermore, we believe that a promising approach to gain understanding on the dynamic processes taking place in urban flows is to perform modal analysis, as discussed in the Appendix. For instance, recent work by Amor et al. [140] has revealed the potential of using dynamic mode decomposition (DMD) to understand the complex physics of the wake in a wall-mounted square cylinder. Other approaches based on convolutional networks and autoencoders [141-143], combined with an adequate modelling of the temporal dynamics [144,145], may pave the way to more reliable flow predictions in urban environments.

\section{Outlook}

In this review we have thoroughly discussed a wide range of available experimental and numerical databases of urban flows, as well as some of the most prominent physical results obtained from them. The very large number of spatio-temporal scales defining turbulent flows motivate researchers to search for new tools to identify the main patterns driving the flow dynamics. There are several methods to extract the main features describing the flow [146], although during the recent years the community has paid special attention to novel data-driven techniques to perform modal decompositions of the flow. Combining the acquired physical knowledge of these techniques with new machine-learning strategies 
it is possible to create fast and efficient reduced-order models (ROMs) accurately modelling the flow [147]. Singular value decomposition (SVD) [148], proper orthogonal decomposition (POD) [149] and dynamic mode decomposition (DMD) [150] are among the most popular techniques generally used for the analysis of various types flows. Their data-driven nature enables their application to a wide range of problems [151-153], and they are powerful tools for the identification of flow structures and the construction of ROMs. Their great success has encouraged researchers to develop more robust variants of these methods, exhibiting accurate results in the analysis of complex flows (turbulent, multi-scale, noise). Among the most popular due to their high efficiency in the analysis of turbulent flows it is important to mention spectral proper orthogonal decomposition (SPOD) [154] and higher-order dynamic mode decomposition (HODMD) [155]. These two techniques combine the benefits of POD with the spectral properties of DMD, providing accurate descriptions of the leading modes in turbulent flows driving the flow dynamics, free of noise and small flow scales [156,157]. More details about the algorithm and the performance of the aforementioned methods is presented in the Appendix, where also some other variants of POD and DMD are briefly introduced. Based on the understanding of two basic concepts, the spectral and spatial flow complexities, defined in Ref. [155] and adapted to the identification of complex flows in Ref. [157], both SPOD and HODMD are suitable tools for the analysis of urban flows. Identifying the main flow patterns with high accuracy and developing high-fidelity ROMs, will shed light into new mechanisms related to the flow behavior, thermal effects and pollutant dispersion in this type of environment.

Author Contributions: The work was ideated by RV and SLC, and the article was written by the three authors.

Funding: This research was funded by the Göran Gustafsson Foundation, and the computer time was provided by the Swedish National Infrastructure for Computing (SNIC).

Acknowledgments: The authors acknowledge the funding provided by the Göran Gustafsson Foundation. The numerical simulations were carried out on resources provided by the Swedish National Infrastructure for Computing (SNIC) at PDC.

Conflicts of Interest: The authors declare no conflict of interest.

\section{Appendix A. Summary of modal decompositions}

The large number of variables and the flow complexity leading the flow dynamics in urban environments, motivate researchers to search for new tools to construct fast and efficient reduced-order models (ROMs). Using data-driven techniques to extract the main features driving the flow, and combining the acquired physical knowledge with new machine-learning strategies to create ROMs is a research topic of high interest that should be explored more in detail. This Appendix presents a brief summary of some relevant data-driven techniques based on modal decompositions exhibiting good performance when analyzing complex flows [151,152,154,156-158] (see more details and other techniques in Refs. $[147,153,159])$. These method are powerful tools that could be potentially used in the construction of new ROMs for urban environments, among others applications.

For convenience, to apply the following data-driven tools, the data are organized into a snapshot matrix, which considers $K$ snapshots evolving in time as:

$$
\boldsymbol{X}=\boldsymbol{V}_{1}^{K}=\left[\boldsymbol{v}_{1}, \boldsymbol{v}_{2}, \ldots, \boldsymbol{v}_{k}, \boldsymbol{v}_{k+1}, \ldots, \boldsymbol{v}_{K-1}, \boldsymbol{v}_{K}\right],
$$

where $v_{k}$ corresponds to the field variable evaluated at time instant $t_{k}$, for simplicity defined as $v_{k}=v\left(t_{k}\right)$. The dimension of the snapshot matrix is $J \times K$, where $J$ represents the spatial degrees of freedom of the analyzed data and $K$ is the number of snapshots, as defined before. The snapshots forming the snapshot matrix do not necessarily require to be equidistant in time for the techniques introduced in §A.1 and §A.2; however, they need to be equidistant in time, with time interval $\Delta t$, for the techniques introduced in §A.3 and §A.4. 
Appendix A.1. Singular value decomposition and proper orthogonal decomposition

Singular value decomposition (SVD) [148] and proper orthogonal decomposition (POD) [149] are well-known techniques that are generally used to create reduced-order models [160] and to identify flow structures and patterns $[161,162]$. The main characteristic of these two techniques are their ability to represent high-dimensional solutions through low-dimensional models, defined by a few SVD/POD modes, which are associated with a high energy content as detailed below.

POD decomposes the flow field $v(x, y, z, t)$ (in fluid dynamics generally the velocity vector) into temporal, $c_{j}(t)$, and spatial orthogonal modes, $\boldsymbol{\Phi}_{j}(x, y, z)$, as:

$$
\boldsymbol{v}(x, y, z, t) \simeq \sum_{j} c_{j}(t) \boldsymbol{\Phi}_{j}(x, y, z),
$$

where the selection of such spatial modes is optimal in terms of the system energy (kinetic energy if the flow field under consideration is the velocity vector).

POD modes can be identified using two types of algorithms, the classical POD method [149], which is based on the covariance of a state vector changing in time, and the snapshot method [148], which is similar to the SVD method. Hence, while POD is the method providing the expansion (A2), SVD can be considered as one of the algorithms providing the POD modes.

Both the classical POD algorithm and the SVD method are based on the definition of the covariance matrix as $\mathbf{C}=\boldsymbol{X} \boldsymbol{X}^{\top}$ (with dimension $J \times J$ ), where $(\cdot)^{\top}$ denotes the matrix transpose and $\boldsymbol{X}$ is the snapshot matrix defined in (A1). By definition, this matrix is symmetric and positive-definite, hence its corresponding eigenvalues and eigenvectors will be real numbers, and the eigenvectors will be orthogonal among them, forming an orthogonal basis of modes.

The classical POD method solves the following eigenvalue problem:

$$
\mathbf{C} \boldsymbol{\Phi}_{j}=\lambda_{j} \boldsymbol{\Phi}_{j}
$$

where the optimal orthogonal modes $\boldsymbol{\Phi}_{j}$ (POD modes) that better approximate a dynamic field, are related with the largest eigenvalues $\lambda_{j}$. In three-dimensional turbulent flows, this method is prohibitively expensive since it is based on solving the eigenvalue problem of the covariance of a state vector changing in time, with dimensions proportional to the spatial degrees of freedom $(J \times J)$. Hence, the snapshot method should be used instead. This method is based on the fact that the most energetic POD modes are the same as those obtained with the transposed covariance matrix as :

$$
\mathbf{C}^{\top} \mathbf{\Psi}_{j}=\lambda_{j} \mathbf{\Psi}_{j} .
$$

The dimensions of $\mathbf{C}^{\top}$ is proportional to the snapshot number from eq. (A1), i.e. $K \times K$ (let us remember that the dimension of $X$ is $J \times K$ ), in fluid dynamics generally being $K<<J$. Moreover, when the snapshot matrix (A1) is composed by velocity vectors, this covarianze matrix contains the variations of the square of the velocity vector, which agrees with the definition of kinetic energy. In such cases, the POD modes are ranked by levels of kinetic energy.

SVD is a type of factorization that captures the directions of a matrix in which vectors can either grow or shrink. These directions are given by the eigenvalues and eigenvectors of a rectangular matrix. Similar to the snapshot method, SVD applied to the snapshot matrix (A1), decomposes the flow field into spatial $(\boldsymbol{W})$ and temporal $(\boldsymbol{T})$ modes and singular values $\boldsymbol{\Sigma}$, as:

$$
V_{1}^{K} \simeq W \Sigma T^{\top},
$$

where $\boldsymbol{W}^{\top} \boldsymbol{W}=\boldsymbol{T}^{\top} \boldsymbol{T}=\mathbf{I}$, with $\mathbf{I}$ being the $N \times N$ unit matrix, and $\boldsymbol{\Sigma}$ is a diagonal matrix composed by the singular values $\left(\sigma_{1}, \cdots, \sigma_{K}\right)$, which are ranked in decreasing order. This method is strongly connected to the previous eigenvalue problems, where $\sigma_{j}^{2}$ correspond to $\lambda_{j}$, the columns of $\boldsymbol{W}$ are the 
POD modes $\boldsymbol{\Phi}_{j}$ (ranked consistently with their corresponding eigenvalues) and the columns of $\boldsymbol{T}$ are the temporal modes $\Psi_{j}$.

The number of SVD (or POD) modes most relevant to describe the flow can be identified using several criteria. Several methodologies are described in Ref. [163], where SVD and POD methods are also known as principal component analysis (PCA). When solving fluid dynamics problems the standard SVD error, estimated for a certain tolerance $\varepsilon_{1}$, defines the most relevant modes in POD analyses based on the singular values as:

$$
\sigma_{N+1} / \sigma_{1} \leq \varepsilon_{1} .
$$

The most relevant modes can then be used to provide low-dimensional approximations of complex flows.

The good performance of POD/SVD algorithms has been shown in the identification of coherent structures in a wide range of applications modelling complex flows $[160,161]$. However, motivated by improving the accuracy of the method to identify patterns in turbulent flows, some authors have developed several variants of the POD algorithm. To name a few: the multi-resolution POD algorithm [164], where before proceeding with the POD analysis of the data, the correlation matrix C is split into the contribution of different flow scales using a filter (preserving symmetry of the correlation) or a Wavelet Transform; the balanced POD [165,166], which is generally used to produced high-fidelity reduced systems combining POD with the balanced truncation in Control Theory [167]; or the spectral proper orthogonal decomposition [154], which is explained more in detail in the following section.

\section{Appendix A.2. Spectral Proper Orthogonal Decomposition}

Spectral proper orthogonal decomposition (SPOD) [154] is an extension of POD recently introduced for analysis and pattern identification in turbulent flows [156,168-170]. This method calculates the POD modes in the frequency domain, combining the good properties of POD with the information contained in a temporal modal expansion as in dynamic mode decomposition (described below).

The algorithm starts from the snapshot matrix (A1), with dimension $J \times K(J$ represents the degrees of freedom of the computational domain and $K$ is the number of snapshots), which is divided into several windows with dimension $J \times K^{\prime}$, where $K^{\prime}<K$. Applying the Fast Fourier Transform (FFT) to each one of such windows, the main frequencies are calculated. A dominant frequency is selected from the multiple solutions in the different windows and the associated modes are used to create a new snapshot matrix. Finally, POD is applied to the new snapshot matrix, reducing the complexity of the data and removing the noise and the spatial redundancies. This method provides low-rank approximations of the solution, free of noise, small flow scales or spurious results. However, application of FFT to reduced groups of data can be computationally expensive, particularly in three-dimensional computational domains where $J$ may be large. A good option could be inverting the order of the steps performed in algorithm. In other words, it is possible to reduce the degrees of freedom from $J$ to $N$ by first applying the POD method. This is the essence of the higher-order dynamic mode decomposition (HODMD) algorithm presented in the following section.

\section{Appendix A.3. Dynamic Mode Decomposition and Higher-Order Dynamic Mode Decomposition}

Dynamic mode decomposition (DMD) [150] is a technique recently introduced to identify flow structures. The method decomposes spatio-temporal data, $v\left(x, y, z, t_{k}\right)$, into Fourier-like modes as:

$$
\boldsymbol{v}\left(x, y, z, t_{k}\right) \simeq \sum_{m=1}^{M} a_{m} \boldsymbol{u}_{m}(x, y, z) e^{\left(\delta_{m}+i \omega_{m}\right) t_{k}}
$$


for $k=1, \cdots, K$, where $\boldsymbol{u}_{m}(x, y, z)$ are the DMD modes weighted with the amplitudes $a_{m}, \omega_{m}$ are their corresponding frequencies and $\delta_{m}$ are their associated growth rates, representing the temporal growth or decay of the modes in time. The DMD algorithm introduced by Schmid [150] is based on the linear relationship between two consecutive snapshots via the linear Koopman operator $\boldsymbol{R}$. Starting from the snapshot matrix (A1), where the snapshots are organized equidistant in time with time step $\Delta t$, the DMD method is defined using the following Koopman assumption:

$$
V_{2}^{K} \simeq R V_{1}^{K-1}
$$

where the matrices $V_{1}^{K-1}$ and $V_{2}^{K}$ contain from the first to the last but one snapshots, and from the second to the last snapshots of the data base, respectively. The Koopman matrix $R$ contains the dynamics of the system, while the DMD frequencies $\omega_{m}$ and growth rates $\delta_{m}$ are the computed eigenvalues and the computed eigenvectors are used to construct DMD modes $\boldsymbol{u}_{m}(x, y, z)$. Recently, Le Clainche \& Vega [155] introduced the higher-order dynamic mode decomposition (HODMD) method, which is an extension of DMD for the analysis of complex flows (turbulence, multi-scale, flows in transitional regime,...) and noisy experimental data. This method combines the Koopman assumption (A8) with the Taknes' delayed embedded theorem [171]. Hence, HODMD relates $d$ time-delayed snapshots (sub-matrices) using the high-order Koopman assumption as:

$$
\boldsymbol{V}_{d+1}^{K} \simeq \boldsymbol{R}_{1} \boldsymbol{V}_{1}^{K-d}+\boldsymbol{R}_{2} \boldsymbol{V}_{2}^{K-(d-1)}+\ldots+\boldsymbol{R}_{d} \boldsymbol{V}_{d}^{K-1},
$$

which can be understood as the window-shift process carried out in power-spectral density (PSD). Finally, as in DMD, the dynamics of the system is contained in the several Koopman operators, $\boldsymbol{R}_{1}, \cdots, \boldsymbol{R}_{d}$, the eigenvalues of which represent the DMD frequencies and growth rates and their eigenvectors are used to construct the DMD modes, as in standard DMD.

The HODMD algorithm proceeds in two steps (see more details in Ref. [155]):

- Step 1: dimension reduction. Applying truncated singular value decomposition (SVD) to the snapshot matrix $V_{1}^{K}$ yields:

$$
V_{1}^{K} \simeq W \Sigma T^{\top} \equiv W \widehat{T}_{1}^{K}, \quad \text { with } \widehat{T}_{1}^{K}=\Sigma T^{\top} .
$$

The matrix $\hat{T}_{1}^{K}$ is the dimension-reduced snapshot matrix. The number of SVD modes retained in this approximation $N$ is defined as the spatial complexity. These modes are selected as in the SVD algorithm presented in §A.1. A (tunable) tolerance $\varepsilon_{1}$ estimates the standard SVD error, as described in eq. (A6).

- Step 2: the DMD-d algorithm for the dimension-reduced snapshots. The high-order Koopman assumption is applied to the reduced snapshot matrix, resulting in:

$$
\hat{\boldsymbol{V}}_{d+1}^{K} \simeq \boldsymbol{R}_{1} \hat{\boldsymbol{V}}_{1}^{K-d}+\boldsymbol{R}_{2} \hat{\boldsymbol{V}}_{2}^{K-(d-1)}+\ldots+\boldsymbol{R}_{d} \hat{\boldsymbol{V}}_{d}^{K-1} .
$$

After some calculations, the several Koopman operators $\boldsymbol{R}_{1}, \cdots, \boldsymbol{R}_{K}$ are grouped into a single matrix, the eigenvalue problem of which provides the DMD modes, frequencies and growth rates that define the DMD expansion (A7). This expansion is sorted in decreasing order of the mode amplitudes and it is further truncated by eliminating the modes such that:

$$
a_{m} / a_{1}<\varepsilon_{2},
$$

for some tunable parameter $\varepsilon_{2}$. The number of modes retained in this expansion, $M$, is called as the spectral complexity. 
When $d=1$ in equation (A11), HODMD reduces to standard DMD, defined as:

$$
\hat{\boldsymbol{V}}_{2}^{K} \simeq \boldsymbol{R}_{1} \hat{\boldsymbol{V}}_{1}^{K-1}
$$

while for $d>1$, HODMD can be understood as the result of applying standard DMD to a set of enlarged snapshots, defined by the delayed snapshots. Hence, HODMD combines the advantages of standard DMD with some consequences of the delayed-embedding theorem by Takens [171].

The spatial and spectral complexities determine the performance of the HODMD algorithm. When the spectral complexity is larger than the spatial complexity, $M>N$, DMD fails (see additional details and examples in Ref. [155]), thus requiring to use the DMD-d algorithm, with $d>1$, to overcome the problem. In complex flows (turbulent, noisy, etc.), the parameter $\varepsilon_{1}$ is selected so as to retain only the large scales in the flow. Hence, at this step the spatial complexity $N$ can be smaller than the spectral complexity $M$, which is generally very large when modelling complex dynamics (see more details in Ref. [172]). Consequently, HODMD is a suitable tool to identify flow structures in noisy experimental data [173], flows in transitional regime [174] and in other complex flows [172,175,176], even with non-equidistant data in time [157], which is one of the limitations of classical DMD methods.

Finally, it is important to mention the spatio-temporal Koopman decomposition (STKD) [177], an extension of HODMD which decomposes the flow as:

$$
\boldsymbol{v}\left(x, y, z_{s}, t_{k}\right) \simeq \sum_{m, b=1}^{M, B} a_{m b} \boldsymbol{u}_{m b}(x, y) e^{\left(\delta_{m b}+i \omega_{m b}\right) t_{k}+\left(v_{m b}+i \beta_{m b}\right) z_{s}}
$$

for $k=1, \cdots, K$, and $s=1, \cdots, S$, with $v_{m b}$ and $\beta_{m b}$ being the spatial growth rates and wavenumbers associated to the spanwise direction. Similarly, the flow can be decomposed along the remaining spatial directions of the analyzed data as:

$$
\boldsymbol{v}\left(x_{i}, y_{l}, z_{s}, t_{k}\right) \simeq \sum_{m, b, p, q=1}^{M, B, P, Q} a_{m b p q} \boldsymbol{u}_{m b p q} e^{\left(\delta_{m b p q}+i \omega_{m b p q}\right) t_{k}+\left(v_{m b p q}+i \beta_{m b p q}\right) z_{s}+\left(\mu_{m b p q}+i \gamma_{m b p q}\right) y_{l}+\left(\sigma_{m b p q}+i \alpha_{m b p q}\right) x_{i}},
$$

for $k=1, \cdots, K, s=1, \cdots, S, l=1, \cdots, L$ and $i=1, \cdots, I$, with $\alpha_{m b p q}, \gamma_{m b p q}$ and $\beta_{m b p q}$ being the spatial wavenumbers and $\sigma_{m b p q}, \mu_{m b p q}$ and $v_{m b p q}$ the corresponding spatial growth rates. Details of the STKD algorithm can be found in Ref. [177]. The essence of the method is to apply the high-order Koopman assumption presented in equation (A11) to the spatial modes (weighted with the singular values and properly re-organized) of the SVD in (A10) from Step 1 in the HODMD algorithm. A novel Koopman operator defines a new eigenvalue problem, the eigenvalues of which represent the spatial growth rates and wavenumbers in the previous expansion. This method has been successfully used to identify the main flow structures in complex flows $[157,172]$ and to identify the flow instabilities leading the flow transition in the wake of a wind turbine [178].

\section{Appendix A.4. Some Variants of DMD not related to time-lagged snapshots}

In recent years, DMD has been used in a wide range of applications in fluid dynamics to identify coherent structures [151] and global instabilities $[179,180]$. The high potential of this tool has motivated several researchers to define new algorithms which widen the type of applications of the tool, providing in some cases more accurate solutions. To mention a few of them:

- Sparsity-promoting DMD [181]. This method uses convex optimization techniques to identify a smaller set of important modes.

- Extended DMD [182]. This algorithm includes more basis functions in the standard DMD approximation, a fact that allows the method to retain more modes, thus enabling the description of more complex dynamical systems. 
- Optimized DMD [183]. An optimization problem is solved to compute the DMD expansion (A7). Both DMD and HODMD are purely linear-algebra-driven approaches compared to optimized DMD. Furthermore, other authors [184] have developed other linear-algebra-oriented algorithms which treat separable nonlinear least-squares problems.

- DMD variants to treat noisy data. Several authors have put an effort on identifying and removing noise from the analyzed data. Among other techniques it is important to mention the method by Dawson et al. [185], which characterizes the noise properties; the total least-squares DMD by Hemati et al. [186], which combines standard DMD with total least squares; and the method by Takeishi et al. [187], which combines standard DMD with a Bayesian formulation.

- Multi-resolution DMD (mrDMD) [188]. The main idea behind mrDMD is to separate the highand low-frequency events which generally occur in complex flows. The algorithm divides the snapshot matrix (A1) into several segments to identify high- and low-frequency modes depending on the segment length. The resulting DMD expansion (A7) is then represented by the various sub-expansions of DMD modes with different frequency ranges. A similar idea is behind the multi-resolution POD algorithm [164].

\section{References}

1. European Commission Urbanisation worldwide. Available at: https://ec.europa.eu/knowledgetpolicy/foresight/ topic/continuing-urbanisation/urbanisation-worldwide_en.

2. United Nations Climate Action. Cities and pollution contribute to climate change. Available at: https: //wwww.un.org/en/climatechange/cities-pollution.shtml.

3. United Nations Paris Agreement on Climate Change. Available at: https://unfccc.int/process-and-meetings/ the-paris-agreement/the-paris-agreement 2015.

4. European Environment Agency. Air quality in Europe - 2019 report. ISSN 1977-8449 2019.

5. Lelieveld, J.; Klingmüller, K.; Pozzer, A.; Pöschl, U.; Fnais, M.; Daiber, A.; Münzel, T. Cardiovascular disease burden from ambient air pollution in Europe reassessed using novel hazard ratio functions. Eur. Heart J. 2019, 40, 1590-1596.

6. Manoli, G.; Fatichi, S.; Schläpfer, M.; Yu, K.; Crowther, T.W.; Meili, N.; Burlando, P.; Katul, G.G.; Bou-Zeid, E. Magnitude of urban heat islands largely explained by climate and population. Nature 2019, 573, 55-60.

7. Heaviside, C.; Vardoulakis, S.; Cai, X.M. Attribution of mortality to the urban heat island during heatwaves in the West Midlands, UK. Environ. Healt 2016, 15, S27.

8. EC Air Quality Framework Directive. European Commission, ambient air quality assessment and management. Council Directive 96/62/EC 1996.

9. Carpentieri, M. Pollutant dispersion in the urban environment. Rev. Environ. Sci. Biotechnol. 2013, 12, 5-8.

10. Weng, Q. Thermal infrared remote sensing for urban climate and environmental studies: Methods, applications, and trends. ISPRS J. Photogramm. 2009, 64, 335-344.

11. UN General Assembly (UNGA). Transforming our world: the 2030 Agenda for Sustainable Development. Resolut. A/RES/70/1, 25, 1-35 2015.

12. Vinuesa, R.; Azizpour, H.; Leite, I.; Balaam, M.; Dignum, V.; Domisch, S. nad Felländer, A.; Langhans, S.D.; Tegmark, M.; Fuso Nerini, F. The role of artificial intelligence in achieving the Sustainable Development Goals. Nat. Commun. 2020, 11, 233.

13. Britter, R.E.; Hanna, S.R. Flow and dispersion in urban areas. Annu. Rev. Fluid Mech. 2003, 35, 469-496.

14. Grimmond, S.B.; Oke, T.R. Turbulent heat fluxes in urban areas: observations and a local-scale urban meteorological parameterization schemes. J. Appl. Meteorol. 2002, 41, 792-810.

15. Oke, T.R. Street design and urban canopy layer climate. Energ. Buildings 1988, 11, 103-112.

16. Sini, J.F.; Anquetin, S.; Mestayer, P.G. Pollutant dispersion and thermal effects in urban street canyons. Atmospheric Environ. 1996, 30, 2659-2677.

17. Vinuesa, R.; Negi, P.S.; Atzori, M.; Hanifi, A.; Henningson, D.S.; Schlatter, P. Turbulent boundary layers around wing sections up to $R e_{c}=1,000,000$. Int. J. Heat Fluid Flow 2018, 72, 86-99.

18. Jeong, J.; Hussain, F. On the identification of a vortex. J. Fluid Mech. 1995, 285, 69-94.

19. Torres, P. High-order spectral simulations of the flow in a simplified urban environment. Bachelor Thesis, Polytechnic University of Valencia, Spain 2020. 
20. Zajic, D.; Fernando, H.J.S.; Calhoun, R.; Princevac, M.; Brown, M.J.; Pardyjak, E.R. Flow and Turbulence in an Urban Canyon. J. Appl. Meteorol. Climatol. 2011, 50, 203-223.

21. Britter, R.E.; Hunt, J.C.R. Velocity measurements and order of magnitude estimates of the flow between two buildings in a simulated atmospheric boundary layer. J. Ind. Aerodyn. 1975, 4, 165-182.

22. Penwarden, A.D.; Wise, A.F.E. Wind environments between buildings. Building Research Establishment Report. Dept. of the Environment, H.M.S.O., London 1975.

23. Isyumov, N.; Davenport, A.G. The ground level wind environment in built-up area. Int. Conf. on Wind Effects on Buildingsand Structure 1977, 4, 165-182.

24. Hlevca, D.; Degeratu, M. Atmospheric boundary layer modeling in a short wind tunnel. Eur. J. Mech. B/Fluids 2020, 79, 367-375.

25. Counihan, J. Adiabatic Atmospheric Boundary Layer: A Review of the Analysis of Data from the Period 1880-1972. Atmos. Environ. 1975, 9, 871-905.

26. Martinuzzi, R.J.; Tropea, C. The flow around surface-mounted, prismatic obstacles placed in a fully developed channel flow. J. Fluids Eng. 1993, 115, 85-92.

27. Cheng, H.; Castro, I.P. Near wall flow over urban-like roughness. Boundary-Layer Meteorol. 2002, 104, 229-259.

28. Castro, I.P.; Cheng, H.; Reynolds, R.T. Turbulence over urban-type roughness: deductions from wind-tunnel measurements. Boundary-Layer Meteorol. 2006, 118, 109-131.

29. Reynolds, R.T.; Castro, I.P. Measurements in an urban-type boundary layer. Exp. Fluids 2008, 45, 141-156.

30. MacDonald, R.; Schofield, S.C.; Slawson, P. Physical modelling of urban roughness using arrays of regular roughness elements. Water Air Soil Pollut. 2002, 2, 541-554.

31. Wang, Z.; Plate, E.; Rau, M.; Keiser, R. Scale effects in wind tunnel modelling. J. Wind Eng. Ind. Aerodyn. 1996, 61, 113-130.

32. Xing, F.; Mohotti, D.; Chauhan, K.A. Experimental and numerical study on mean pressure distributions around an isolated gable roof building with and without openings. Build. Environ. 2018, 132, 30-44.

33. Martinuzzi, R.; Havel, B. Vortex shedding from two surface-mounted cubes in tandem. Int. J. Heat Fluid Flow 2004, 25, 364-372.

34. Hussein, H.; Martinuzzi, R. Energy balance for turbulent flow around a surface mounted cube placed in a channel. Phys. Fluids 1996, 8, 764-780.

35. Nagib, H.M.; Corke, T.C. Wind microclimate around buildings: characteristics and control. J. Wind Eng. Ind. Aerodyn. 1984, 16, 1-15.

36. Vinuesa, R.; Schlatter, P.; Malm, J.; Mavriplis, C.; Henningson, D.S. Direct numerical simulation of the flow around a wall-mounted square cylinder under various inflow conditions. J. Turbul. 2015, 16, 555-587.

37. Rotach, M.W.; Fisher, B.; Piringer, M. COST 715 workshop on urban boundary layer parameterizations. $B$. Am. Meteorol. Soc. 2002, 83, 1501-1504.

38. Coceal, O.; Belcher, S. A canopy model of mean winds through urban areas. Q. J. R. Meteorol. Soc. 2004, 130, 1349-1372.

39. Rafailidis, S. Influence of building areal density and roof shape on the wind characteristics above a town. Boundary-Layer Meteorol. 1997, 85, 255-271.

40. Kastner-Klein, P.; Rotach, M. Mean flow and turbulence characteristics in an urban roughness sublayer. Boundary-Layer Meteorol. 2004, 111, 55-84.

41. Li, X.; Leung, D.; Liu, C.; Lam, K. Physical modeling of flow field inside urban street canyons. J. Appl. Meteorol. 2008, 47, 2058-2067.

42. Princevac, M.; Baik, J.; Li, X.; Pan, H.; Park, S. Lateral channeling within rectangular arrays of cubical obstacles. J. Wind Eng. Ind. Aerodyn. 2010, 98, 377-385.

43. Richards, P.; Hoxey, R.; Short, L. Wind pressures on a $6 \mathrm{~m}$ cube. J. Wind Eng. Ind. Aerodyn. 2001, 89, 1553-1564.

44. Richards, P.; Hoxey, R.; Connell, B.; Lander, D. Wind-tunnel modelling of the Silsoe Cube. J. Wind Eng. Ind. Aerodyn. 2007, 95, 1384-1399.

45. Takimoto, H.; Sato, A.; Onomaura, S.; Barlow, J.; Moriwaki, R.; Inagaki, A.; Kanda, M. Particle image velocimetry measurements of turbulent flow within outdoor and indoor urban scale models and flushing motions in urban canopy layers. Boundary-Layer Meteorol. 2011, 140, 295-314. 
46. Hagishima, A.; Tanimoto, J.; Nagayama, K. Aerodynamic parameters of regular arrays of rectangular blocks with various geometries. Boundary-Layer Meteorol. 2009, 132, 315-337.

47. Kovar-Panskus, A.; Louka, P.; Sini, J.; Savory, E.; Czech, M.; Abdelqari, A.; Mestayer, P.; Toy, N. Influence of geometry on the mean flow within urban street canyons - A comparison of wind tunnel experiments and numerical simulations. Water Air Soil Pollut. 2002, 2, 365-380.

48. Kovar-Panskus, A.; Moulinneuf, L.; Savory, E.; Abdelqari, A.; Sini, J.F.; Rosant, J.M.; Robins, A.; Toy, N. A wind tunnel investigation of the influence of solar-induced wall-heating on the flow regime within a simulated urban street canyon. Water Air Soil Pollut. 2002, 2, 555-571.

49. Gromke, C.; Ruck, B. Influence of trees on the dispersion of pollutants in an urban street canyon Experimental investigation of the flow and concentration field. Atmos. Environ. 2007, 41, 3287-3302.

50. Gromke, C.; Ruck, B. On the impact of trees on dispersion processes of traffic emissions in street canyons. Boundary-Layer Meteorol. 2009, 131, 19-34.

51. Gromke, C.; Buccolieri, R.; Sabatino, S.D.; Ruck, B. Dispersion study in a street canyon with tree planting by means of wind tunnel and numerical investigations - Evaluation of CFD data with experimental data. Atmos. Environ. 2008, 42, 8640-8650.

52. Gayev, Y.; Savory, E. Influence of street obstructions on flow processes within urban canyons. J. Wind Eng. Ind. Aerodyn. 1999, 82, 89-103.

53. Kellnerova., R.; Kukacka, J.; Jurcakova, K.; Uruba, V.; Janur, Z. PIV measurement of turbulent flow within a street canyon: detection of coherent motion. J. Wind Eng. Ind. Aerodyn. 2012, 104-106, 302-313.

54. Fernando, H.J.S.; Zajic, D.; Di Sabatino, S.; Dimitrova, R.; Hedquist, B.; Dallman, A. Flow, turbulence, and pollutant dispersion in urban environments. Phys. Fluids 2010, 22, 051301.

55. Biltoft, C.A. Customer report for Mock Urban Setting Test. DPG Document No WDTC-FR-01-121, West Desert Test Center, US Army Dugway Proving Ground, Dugway, Utah 2001.

56. Sharma, A.; Fernando, H.J.S.; Hellmann, J.; Chen, F. Sensitivity of WRF model to urban parametrizations, with applications to Chicago metropolitan urban heat island. 4th Joint US-European Fluids Engineering Summer Meeting FEDSM14 2014.

57. Di Sabatino, S.; Leo, L.S.; Hedquist, B.C.; Carter, W.; Fernando, H.J.S. Results from the Phoenix Urban Heat Island (UHI) experiment: effects at the local, neighbourhood and urban scales. Eighth Symposium on the Urban Environment, Phoenix, Arizona (USA) 2009.

58. Vita, G.; Shu, Z.; Jesson, M.; Quinn, A.; Hemida, H.; Sterling, M.; Baker, C. On the assessment of pedestrian distress in urban winds. J. Wind. Eng. Ind. Aerodyn. 2020, 203, 1-18.

59. C. Hirose, C.; Ikegaya, N.; Hagishima, A.; Tanimoto, J. Outdoor measurement of wall pressure on cubical scale model affected by atmospheric turbulent flow. Build. Environ. 2019, 160, 1-11.

60. Vinuesa, R.; Nagib, H.M. Enhancing the accuracy of measurement techniques in high Reynolds number turbulent boundary layers for more representative comparison to their canonical representations. Eur. J. Mech.-B/Fluids 2016, 55, 300-312.

61. Vinuesa, R.; Schlatter, P.; Nagib, H.M. Role of data uncertainties in identifying the logarithmic region of turbulent boundary layers. Exp. Fluids 2014, 55, 1751.

62. Vinuesa, R. Synergetic computational and experimental studies of wall-bounded turbulent flows and their two-dimensionality. PhD Thesis, Illinois Institute of Technology, Chicago (USA) 2013.

63. Örlü, R.; Vinuesa, R. Thermal anemometry. Experimental Aerodynamics. S. Discetti and A. Ianiro (editors). CRC Press Taylor \& Francis Group, Boca Raton, FL 2017.

64. Gadilhe, A.; Janvier, L.; Barnaud, G. A wind tunnel study of effects of twisted wind flows on the pedestrian-level wind field in an urban environment. J. Wind. Eng. Ind. Aerodyn. 1993, 46, 755-766.

65. Weerasuriya, A.U.; Tse, K.T.; Zhang, X.; Li, S.W. A wind tunnel study of effects of twisted wind flows on the pedestrian-level wind field in an urban environment. Build. Environ. 2018, 128, 225-235.

66. Chauhan, K.A.; Monkewitz, P.A.; Nagib, H.M. Criteria for assessing experiments in zero pressure gradient boundary layers. Fluid Dyn. Res. 2009, 41, 021404.

67. Sanmiguel Vila, C.; Vinuesa, R.; Discetti, S.; Ianiro, A.; Schlatter, P.; Örlü, R. On the identification of well-behaved turbulent boundary layers. J. Fluid Mech. 2017, 822, 109-138.

68. Mo, Z.; Liu, C.H. Wind tunnel measurements of pollutant plume dispersion over hypothetical urban areas. Build. Environ. 2018, 132, 357-366. 
69. Talluru, K.M.; Philip, J.; Chauhan, K.A. Self-similar spectra of point-source scalar plumes in a turbulent boundary layer. J. Fluid Mech. 2019, 870, 698-717.

70. Hertwig, D.; Grimmond, S.; Kotthaus, S.; Vanderwel, C.; Gough, H.; Haeffelin, M.; Robins, A. Variability of physical meteorology in urban areas at different scales: implications for air quality. Faraday Discussions. Royal Society of Chemistry 2020, pp. 2-11.

71. Monnier, B.; Neiswander, B.; Wark, C. Stereoscopic particle image velocimetry measurements in an urban-type boundary layer: insight into flow regimes and incidence angle effect. Boundary-Layer Meteorol. 2010, 135, 243-268.

72. Nagib, H.; Morkovin, M.; Yung, J.; Tan-Atichat, J. On modeling of atmospheric surface layers by the counter-jet technique. AIAA J. 1974, 14(2), 185-190.

73. Ferreira, M.; Ganapathisubramani, B. PIV-based pressure estimation in the canopy of urban-like roughness. Exp. Fluids 2020, 61, 70.

74. Michioka, T.; Takimoto, H.; Sato, A. Large-Eddy Simulation of pollutant removal from a three-dimensional street canyon. Boundary-Layer Meteorol. 2014, 150, 259-275.

75. Boppana, V.B.L.; Xie, Z.T.; Castro, I.P. Thermal stratification effects on flow over a generic urban canopy. Boundary-Layer Meteorol. 2014, 130, 141-162.

76. Tong, H.; Walton, A.; Sang, J.; Chan, J. Numerical simulation of the urban boundary layer over the complex terrain of Hong Kong. Atmos. Environ. 2005, 39), 3549-3563.

77. Santiago, J.; Dejoan, A.; Martilli, A.; Martin, F.; Pinelli, A. Comparison Between Large-Eddy Simulation and Reynolds-Averaged Navier-Stokes Computations for the MUST Field Experiment. Part I: Study of the Flow for an Incident Wind Directed Perpendicularly to the Front Array of Containers. Boundary-Layer Meteorol. 2010, 135, 109-132.

78. Jacob, J.; Sagaut, P. Wind comfort assessment by means of large eddy simulation with lattice Boltzmann method in full scale city area. Building Environ. 2019, 139), 110-124.

79. Sullivan, P.; J.C., W.; Patton, E.; Jonker, H.; Mironov, D. Turbulent Winds and Temperature Fronts in Large-Eddy Simulations of the Stable Atmospheric Boundary Layer. J. Atmos. Sci. 2016, 73(4), 1815-1840.

80. Dejoan, A.; Santiago, J.; Martilli, A.; Martin, F.; Pinelli, A. Comparison Between Large-Eddy Simulation and Reynolds-Averaged Navier-Stokes Computations for the MUST Field Experiment. Part II: Effects of Incident Wind Angle Deviation on the Mean Flow and Plume Dispersion. Boundary-Layer Meteorol. 2010, 133), 135-150.

81. Nakayama, H.; Takemi, T.; H., N. LES Analysis of the Aerodynamic Surface Properties for Turbulent Flows over Building Arrays with Various Geometries. J. Appl. Meteorol. Clim. 2011, 50(8), 1692-1712.

82. Nakayama, H.; Takemi, T.; H., N. Large-eddy simulation of urban boundary-layer flows by generating turbulent inflows from mesoscale meteorological simulations. Atmos. Sci. Let. 2012, 13, 180-186.

83. Smagorinsky, J. General circulation experiments with the primitive equations. Mon. Weather Rev. 1963, 91(3), 51-78.

84. Kataoka, H.; Mizuno, M. Numerical flow computation around aeroelastic 3D square cylinder using inflow turbulence. Wind Struct. 2002, 5, 379-392.

85. Giometto, M.; Christen, A.; Meneveau, C.; Fang, J.and Krafczyk, M.; Parlange, M. Spatial Characteristics of Roughness Sublayer Mean Flow and Turbulence Over a Realistic Urban Surface. Boundary-Layer Meteorol. 2016, 160, 425-452.

86. Mason, P.; Thomson, D. Stochastic backscatter in large-eddy simulations of boundary layers. J. Fluid Mech. 1992, 242, 51-78.

87. Bou-Zeid, E.; Meneveau, C.; Parlange, M. A scale-dependent Lagrangian dynamic model for large eddy simulation of complex turbulent flows. Phys. Fluids 2005, 17(2), 25-105.

88. Inagaki, A.; Kanda, M.; Ahmad, N.; Yaki, A.; Onodera, N.; Aoki, T. A Numerical Study of Turbulence Statistics and the Structure of a Spatially-Developing Boundary Layer Over a Realistic Urban Geometry. Boundary-Layer Meteorol. 2017, 164, 161-181.

89. Grylls, T.; Suter, I.; van Reeuwijk, M. Steady-State Large-Eddy Simulations of Convective and Stable Urban Boundary Layers. Boundary-Layer Meteorol. 2020, 175, 309-341.

90. Belcher, S. Mixing and transport in urban areas. Philos. Trans. R Soc. 2005, 363, 3583-3597.

91. Theurer, W.; Plate, E.; Hoeschele, K. Semi-empirical models as a combination of wind tunnel and numerical dispersion modelling. Atmos. Environ. 1996, 30, 3583-3597. 
92. Davidson, M.; Snyder, W.; Lawson, R.; Hunt, J. Wind tunnel simulations of plume dispersion through groups of obstacles. Atmos. Environ. 1996, 30, 3715-3725.

93. Macdonald, R.; Griffiths, R.; Cheah, S. Field experiments of dispersion through regular arrays of cubic structures. Atmos. Environ. 1997, 31, 783-795.

94. Coceal, O.; Thomas, T.G.; Castro, I.; Belcher, S. Mean flow and turbulence statistics over groups of urban-like cubical obstacles. Boundary-Layer Meteorol. 2006, 121, 491-519.

95. Coceal, O.; Dobre, A.; Thomas, T.; Belcher, S. Structure of turbulent flow over regular arrays of cubical roughness. J. Fluid Mech. 2007, 589, 375-409.

96. Brandford, S.; Coceal, O.; Thomas, T.; Belcher, S. Dispersion of a Point-Source Release of a Passive Scalar Through an Urban-Like Array for Different Wind Directions. Boundary-Layer Meteorol. 2011, 139, 367-394.

97. Germano, M.; Piomelli, U.; Moin, P.; Cabot, W. A dynamic subgrid-scale eddy viscosity model. Phys. Fluids 1991, A3, 1760-1766.

98. Lilly, D. A proposed modification of the Germano subgrid-scale closure method. Phys. Fluids 1992, A4, 633-636.

99. Lee, J.; Sung, H.; Krogstad, P. Direct numerical simulation of the turbulent boundary layer over a cube-roughened wall. J. Fluid Mech. 2011, 669, 397-431.

100. Cheng, W.C.; Porté-Agel, F. Adjustment of turbulent boundary-layer flow to idealized urban surfaces: a large-eddy simulation study. Boundary-Layer Meteorol. 2015, 155, 249-270.

101. Tomas, J.M.; Pourquie, M.J.B.M.; Jonker, H.J.J. Stable stratification effects on flow and pollutant dispersion in boundary layers entering a generic urban environment. Boundary-Layer Meteorol. 2016, 159, 221-239.

102. Vreman, A.W. An eddy-viscosity subgrid-scale model for turbulent shear flow: algebraic theory and applications. Phys. Fluis 2004, 16, 3670.

103. Tomas, J.M.; Eisma, H.E.; Pourquie, M.J.B.M.; Elsinga, G.E.; Jonker, H.J.J.; Westerweel, J. Pollutant dispersion in boundary layers exposed to rural-to-urban transitions: varying the spanwise length scale of the roughness. Boundary-Layer Meteorol. 2017, 163, 225-251.

104. Eisma, H.E.; Tomas, J.M.; Pourquie, M.J.B.M.; Elsinga, G.E.; Jonker, H.J.J.; Westerweel, J. Effects of a fence on pollutant dispersion in a boundary layer exposed to a rural-to-urban transition. Boundary-Layer Meteorol. 2018, 169, 185-208.

105. Vinçont, J.; Simoëns, S.; Ayrault, M.; Wallace, J.M. Passive scalar dispersion in a turbulent boundary layer from a line source at the wall and downstream of an obstacle. J. Fluid Mech. 2000, 424, 127-167.

106. Soulhac, L. Modelisation de la dispersion atmospheric a l'interieur de la canopee urbaine. PhD thesis, Ecole Centrale de Lyon 2000.

107. Soulhac, L.; Salizzoni, P.; Cierco, F.; Perkins, R. The model SIRANE for atmospheric urban pollution dispersion: part I, presentation of the model. Atmos. Environ. 2011, 45(39), 7379-7395.

108. Hamlyn, D.; Hilderman, T.; Britter, R. A simple network approach to modelling dispersion among large groups of obstacle. Atmos. Environ. 2007, 41, 5848-5862.

109. Belcher, S.; Coceal, O.; Goulart, E.; Rudd, A.; Robins, A. Processes controlling atmospheric dispersion through city centres. J. Fluid Mech. 2015, 763, 51-81.

110. Goulart, E.; Coceal, O.; Belcher, S. Dispersion of a passive scalar within and above an urban street network. Boundary-Layer Meteorol. 2018, 166, 351-366.

111. Hutchins, N.; Chauhan, K.; Marusic, I.; Monty, J.; Klewicki, J. Towards reconciling the large-scale structure of turbulent boundary layers in the atmosphere and laboratory. Boundary-Layer Meteorol. 2012, 145, 273-306.

112. Choi, H.; Moin, P. Grid-point requirements for large eddy simulation: Chapman's estimates revisited. Phys. Fluids 2012, 24, 011702.

113. Businger, J.A.; Wyngaard, J.C.; Izumi, Y.; Bradley, E.F. Flux-profile relationships in the atmospheric surface layer. J. Atmos. Sci. 1971, 28, 181-189.

114. Giometto, M.G.; Christen, A.; Meneveau, C.; Fang, J.; Krafczyk, M.; Parlange, M.B. Spatial characteristics of roughness sublayer mean flow and turbulence over a realistic urban surface. Boundary-Layer Meteorol. 2016, 160, 425-452.

115. Rotach, M.W.; Vogt, R.; Bernhofer, C.; Batchvarova, E.; Christen, A.; Clappier, A.; Feddersen, B.; Gryning, S.E.; Martucci, G.; Mayer, H.; Mitev, V.; Oke, T.R.; Parlow, E.; Richner, H.; Roth, M.; Roulet, Y.A.; Ruffieux, D.; Salmond, J.A.; Schatzmann, M.; Voogt, J.A. BUBBLE - An urban boundary layer meteorology project. Theor. Appl. Climatol. 2005, 81, 231-261. 
116. Bou-Zeid, E.; Meneveau, C.; Parlange, M. A scale-dependent Lagrangian dynamic model for large eddy simulation of complex turbulent flows. Phys. Fluids 2005, 17, 025105.

117. Moeng, C. A large-eddy-simulation model for the study of planetary boundary-layer turbulence. J. Atmos. Sci. 1984, 13, 2052-2062.

118. Dogan, E.; Örlü, R.; Gatti, D.; Vinuesa, R.; Schlatter, P. Quantification of amplitude modulation in wall-bounded turbulence. Fluid Dyn. Res. 2019, 51, 011408.

119. Schenk, F.; Vinuesa, R. Enhanced large-scale atmospheric flow interaction with ice sheets at high model resolution. Results Eng. 2019, 3, 100030.

120. Schenk, F.; Väliranta, M.; Muschitiello, F.; Tarasov, L.; M..; Heikkilä, M.; Björck, S.; Brandefelt, J.; Johannson, A.; Näslund, J.O.; Wohlfarth, B. Warm summers during the Younger Dryas cold reversal. Nat. Commun. 2018, 9, 1634.

121. Hunt, J.C.R.; Abell, C.J.; Peterka, J.A.; Woo, H. Kinematical studies of the flows around free or surface-mounted obstacles: applying topology to flow visualization. J. Fluid Mech. 1978, 86, 179-200.

122. Vinuesa, R.; Prus, C.; Schlatter, P.; Nagib, H.M. Convergence of numerical simulations of turbulent wall-bounded flows and mean cross-flow structure of rectangular ducts. Meccanica 2016, 51, 3025-3042.

123. Sousa, J. Turbulent flow around a surface-mounted obstacle using 2D-3C DPIV. Exp. Fluids 2002, $33,854-862$.

124. Zhou, J.; Adrian, R.J.; Balachandar, S.; Kendali, T.M. Mechanisms for generating coherent packets of hairpin vortices. J. Fluid Mech. 1990, 387, 353-396.

125. Becker, S.; Lienhart, H.; Durst, F. Flow around three-dimensional obstacles in boundary layers. J. Wind Eng. Ind. Aerodyn. 2002, 90, 265-279.

126. Martinuzzi, R. Experimentelle Untersuchungen der Umströmung wandgebundener, rechteckiger, prismatischer Hindernisses. Dissertation Thesis, Universität Erlangen-Nürnberg 1982.

127. Kim, J.; Baik, J. A numerical study of the effects of ambient wind direction on flow and dispersion in urban street canyons using the RNG $k-\varepsilon$ turbulence model. Atmos. Environ. 2004, 38, 3039-3048.

128. Kawai, H.; Okuda, Y.; Ohashi, M. Near wake structure behind a 3D square prism with the aspect ratio of 2.7 in a shallow boundary layer flow. J. Wind Eng. Ind. Aerodyn. 2012, 104-106, 196-202.

129. del Álamo, J.C.; Jiménez, J.; Zandonade, P.; Moser, R.D. Self-similar vortex clusters in the turbulent logarithmic region. J. Fluid Mech. 2006, 561, 329-358.

130. Lozano-Durán, A.; Flores, O.; Jiménez, J. The three-dimensional structure of momentum transfer in turbulent channels. J. Fluid Mech. 2012, 694, 100-130.

131. Atzori, M.; Vinuesa, R.; Lozano-Durán, A.; Schlatter, P. Characterization of turbulent coherent structures in square duct flow. J. Phys.: Conf. Ser. 2018, 1001, 012008.

132. Atzori, M.; Vinuesa, R.; Lozano-Durán, A.; Schlatter, P. Coherent structures in turbulent boundary layers over an airfoil. J. Phys.: Conf. Ser. 2020, 1522, 012020.

133. Hunt, J.C.R.; Wray, A.A.; Moin, P. Eddies, streams, and convergence zones in turbulent flows. Center for Turbulence Research (CTR) Proceedings of Summer Program 1998.

134. Krajnovic, S.; Davidson, L. Flow around a three-dimensional bluff body. In: 9th international symposium on flow visualization, Heriot-Watt Univeristy, Edinburgh 2000.

135. Chong, M.S.; Perry, A.E.; Cantwell, B.J. A general classification of three-dimensional flow fields. Phys. Fluids 1990, 5, 765.

136. Kida, S.; Miura, H. Identification and analysis of vortical structures. Eur. J. Mech. B Fluids 1998, 17, 471-488.

137. Graftieaux, L.; Michard, M.; Grosjean, N. Combining PIV, POD and vortex identification algorithms for the study of unsteady turbulent swirling flows. Meas. Sci. Technol. 2001, 12, 1422-1429.

138. Monnier, B.; Goudarzi, S.A.; Vinuesa, R.; Wark, C. Turbulent structure of a simplified urban fluid flow studied through stereoscopic particle image velocimetry. Boundary-Layer Meteorol. 2018, 166, 239-268.

139. Rempfer, D.; Wark, C.; Monnier, B.; Kandala, S. Characterization and low-dimensional modeling of urban fluid flow. AFOSR Final Report, contract number FA9550-11-1-0056 2014.

140. Amor, C.; Pérez, J.M.; Schlatter, P.; Vinuesa, R.; Le Clainche, S. Soft computing techniques to analyze the turbulent wake of a wall-mounted square cylinder. Advances in Intelligent Systems and Computing 2019, 950, 577-586. 
141. Guastoni, L.; Güemes, A.; Ianiro, A.; Discetti, S.; Schlatter, P.; Azizpour, H.; Vinuesa, R. Convolutional-network models to predict wall-bounded turbulence from wall quantities. arXiv preprint arXiv:2006.12483 2020.

142. Murata, T.; Fukami, K.; Fukagata, K. Nonlinear mode decomposition with convolutional neural networks for fluid dynamics. J. Fluid Mech. 2020, 882, A13.

143. Eivazi, H.; Veisi, H.; Naderi, M.H.; Esfahanian, V. Deep neural networks for nonlinear model order reduction of unsteady flows. arXiv preprint arXiv:2007.00936 2020.

144. Srinivasan, P.A.; Guastoni, L.; Azizpour, H.; Schlatter, P.; Vinuesa, R. Predictions of turbulent shear flows using deep neural networks. Phys. Rev. Fluids 2019, 4, 054603.

145. Eivazi, H.; Guastoni, L.; Schlatter, P.; Azizpour, H.; Vinuesa, R. Recurrent neural networks and Koopman-based frameworks for temporal predictions in turbulence. arXiv preprint arXiv:2005.02762 2020.

146. Haller, G. An objective definition of a vortex. J. Fluid Mech. 2005, 525, 1-26.

147. Le Clainche, S. An Introduction to Some Methods for Soft Computing in Fluid Dynamics. International Workshop on Soft Computing Models in Industrial and Environmental Applications, Springer 2019, pp. 557-566.

148. Sirovich, L. Turbulence and the dynamics of coherent structures. Parts I-III. Quarterly of applied mathematics 1987, 45(3), 561-571.

149. Lumley, J.L. The Structure of Inhomogeneous Turbulent Flows. Proceedings of the International Colloquium on the Fine Scale Structure of the Atmosphere and Its Influence on Radio Wave Propagation, edited by A. M. Yaglam, and V. I. Tatarsky, Doklady Akademii Nauk SSSR, Nauka, Moscow 1967.

150. Schmid, P. Dynamic mode decomposition of numerical and experimental data. J. Fluid Mech. 2010, $656,5-28$.

151. Schmid, P. Application of the dynamic mode decomposition to experimental data. Exp. in Fluids 2011, 50(4), 1123-1130.

152. Tu, J.; Rowley, C.; Luchtemburg, D.; Brunton, S.; Kutz, J. On dynamic mode decomposition: Theory and applications. J. Comp. Dyn. 2014, 1(2), 391-421.

153. Rowley, C.; Dawson, S. Model reduction for flow analysis and control. Annual Rev. Fluid Mech. 2017, 49, 387-417.

154. Schmidt, O.; Towne, A.; Colonius, T.; Cavalieri, A.; Jordan, P.; Bres, G. Wavepackets and Trapped Acoustic Modes in a Mach 0.9 Turbulent Jet: A Global Stability Analysis. International Workshop on Soft Computing Models in Industrial and Environmental Applications, Springer 2017, 825, 1153-1181.

155. Le Clainche, S.; Vega, J.M. Higher order dynamic mode decomposition. SIAM J. Appl. Dyn. Sys. 2017, 16 (2), 882-925.

156. Geoga, C.; Haley, C.; Siegel, A.; M., A. Frequency-wavenumber Spectral analysis of spatio-temporal flows. J. Fluid Mech. 2018, 848, 545-559.

157. Le Clainche, S.; Izvassarov, D.; Rosti, M.; Brandt, L.; Tammisola, O. Coherent structures in the turbulent flow of an elastoviscoplastic fluid. J. Fluid Mech. 2020, 888, A5.

158. Le Clainche, S.; Vega, J. Analyzing Nonlinear Dynamics via Data-Driven Dynamic Mode Decomposition-Like Methods. Complexity 2018, 6920783, 1-21.

159. Rowley, C.; Dawson, S. Modal analysis of fluid flows: An overview. AIAA J. 2017, pp. 4013-4041.

160. Noack, B.R.; Morzynski, M.; Tadmor, G. Reduced-Order Modelling for Flow Control. Springer, New York 2011.

161. Tammisola, O.; Juniper, M.P. Coherent structures in a swirl injector at $R e=4800$ by nonlinear simulations and linear global modes. J. Fluid Mech., pp. 620-657.

162. Le Clainche, S.; Li, I.; Theofilis, V.; Soria, J. Flow around a hemisphere-cylinder at high angle of attack and low Reynolds number. Part I: Experimental and numerical investigation. Aerosp. Sci. Tech. 2015, 44, 77-87.

163. Parente, A. Experimental and numerical investigation of advanced systems for hydrogen-based fuel compustion. PhD Thesis, University of Pisa 2008.

164. Mendez, M.A.; Balabane, M.; Buchlin, J. Multi-scale proper orthogonal decomposition of complex fluid flows. J. Fluid Mech. 2019, 870, 988-1036.

165. Willcox, K.; Peraire, J. Balanced model reduction via theproper orthogonal decomposition. AIAA J. 2002, 40(11), 2323-2330. 
166. Rowley, C. Model reduction for fluids, using balanced proper orthogonal decomposition. Int. J. Bifurcation Chaos 2005, 15(03), 997.

167. Phillips, J.; Silveira, L. Computer-Aided Design of Integrated Circuits andSystems. IEEE Transactions 2005, 24(1), 43-55.

168. Karami, S.; Soria, J. Analysis of coherent structures in an under-expanded supersonic impinging jet using spectral proper orthogonal decomposition (SPOD). Aerospace 2018, 5(3), 73.

169. Abreu, L.I.; Cavalieri, A.V.G.; Schlatter, P.; Vinuesa, R.; Henningson, D.S. SPOD and resolvent analysis of near-wall coherent structures in turbulent pipe flows. J. Fluid Mech. 2020, 900, A11.

170. Abreu, L.I.; Cavalieri, A.V.G.; Schlatter, P.; Vinuesa, R.; Henningson, D.S. Resolvent modelling of near-wall coherent structures in turbulent channel flow. Int. J. Heat Fluid Flow 2020, 85, 108662.

171. Takens, F. Detecting strange attractors in turbulence. Lecture Notes in Mathematics, D.A. Rand and L.-S. Young. eds., Springer-Verlag 1981, pp. 366-381.

172. Le Clainche, S.; Rosti, M.; Brandt, L. Flow structures and shear-stress predictions in the turbulent channel flow over an anisotropic porous wall. J. Phys. Conf. Series 2020, 1522, 012016.

173. Le Clainche, S.; Vega, J.M.; Soria, J. Higher order dynamic mode decomposition of noisy experimental data: the flow structure of a zero-net-mass-flux jet. Exp. Therm. Fluid Sci. 2017, 88, 336-353.

174. Le Clainche, S.; Vega, J.M. Higher order dynamic mode decomposition to identify and extrapolate flow patterns. Phys. Fluids 2017, 29 (8), 084102.

175. Le Clainche, S.; Ferrer, E. A reduced order model to predict transient flows around straight bladed vertical axis wind turbines. Energies 2018, 11, 566-578.

176. Le Clainche, S.; Moreno-Ramos, R.; Taylor, P.; Vega, J.M. A new robust method to study flight flutter testing. J. Aircraft 2018.

177. Le Clainche, S.; Vega, J.M. Spatio-temporal Koopman decomposition. J. Nonlin. Sci. 2018, 28 (3), 1-50.

178. Le Clainche, S.; Mao, X.; Vega, J.M. New method to capture traveling waves in flow passing a wind turbine. Wind Energy 2019, 22(7), 1095-4244.

179. Rowley, C.; Mezić, I.; Bagheri, S.; Schlatter, P.; Henningson, D. Spectral analysis of nonlinear flows. J. Fluid Mech. 2009, 641, 115-127.

180. Gómez, F.; Clainche, S.L.; Paredes, P.; Hermanns, M.; Theofilis, V. Four decades of studying global linear instability. AIAA J. 2012, 50(12), 2731-2743.

181. Jovanovic, M.; Schmid, P.; Nichols, J. Sparsity-promoting dynamic mode decomposition. Phys. Fluids 2014, 26,024103 .

182. Williams, M.; Kevrekidis, I.; Rowley, C. A data driven approximation of the Koopman operator: extending dynamic mode decomposition. J. Nonlin. Sci. 2015, 25, 1307-1346.

183. Chen, K.; Tu, J.; Rowley, C. Variants of dynamic mode decomposition: boundary condition, Koopman and Fourier analyses. J. Nonlin. Sci. 2012, 22, 8871-8875.

184. Askham, T.; Kutz, J.N. Variable projection methods for an optimized dynamic mode decomposition. SIAM J. Appl. Dyn. Systems 2018, 17, 380-416.

185. Dawson, S.; Hemati, M.; Williams, M.; Rowley, C. Characterizing and correcting for the effect of sensor noise in the dynamic mode decomposition. Exp. Fluids 2016, 17, 42-57.

186. Hemati, M.; Rowley, C.; Deem, E.; Cattafesta, L. De-Biasing the dynamic mode decomposition for applied Koopman spectral analysis of noisy datasets. Theor. Comp. Fluid Dyn., Springer-Verlag 2017, pp. 349-368.

187. Takeishi, N.; Kawahara, Y.; Tabei, Y.; Yairi, T. Bayesian dynamic mode decomposition. Proc. of the 26th Intl Joint Conf. on Artificial Intelligence (IJCAI) 2017, pp. 2814-2821.

188. Kutz, J.; Fu, X.; Brunton, S. Multiresolution Dynamic Mode Decomposition. SIAM J. Appl. Dyn. Sys. 2016, 15(2), 713-735. 